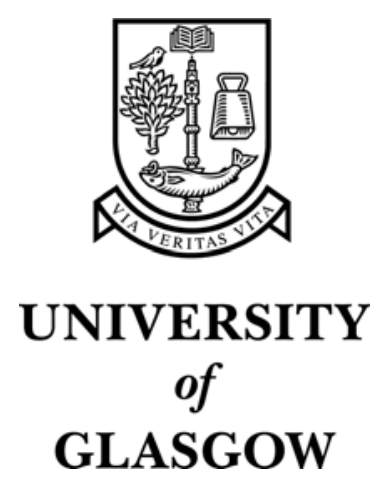

Coton, F.N. and Galbraith, R.A.McD. and Green, R.B. (2005) Analysis of model rotor blade pressures during parallel interaction with twin vortices. Journal of Aircraft 42(6):pp. 1553-1565.

http://eprints.gla.ac.uk/3382/ 


\section{Analysis of Model Rotor Blade Pressures during Parallel Interaction with Twin Vortices}

By

F.N. Coton*, R.B. Green ${ }^{\dagger}$, R.A.McD. Galbraith ${ }^{\ddagger}$

University of Glasgow, Glasgow, G12 8QQ

U.K.

* Professor of Low Speed Aerodynamics, Department of Aerospace Engineering

$\dagger$ Senior Lecturer, Department of Aerospace Engineering

\$ Shoda Professor of Aerospace Systems, Department of Aerospace Engineering 


\section{ABSTRACT}

This paper presents and provides analysis of unsteady surface pressures measured on a model rotor blade as the blade experienced near parallel blade vortex interaction with a twin vortex system. To provide a basis for analysis, the vortex system was characterized by hot-wire measurements made in the interaction plane but in the absence of the rotor. The unsteady pressure response resulting from a single vortex interaction is then presented to provide a frame of reference for the twin vortex results. A series of twin vortex interaction cases are then presented and analyzed. It is shown that the unsteady blade pressures and forces are very sensitive to the inclination angle and separation distance of the vortex pair. When the vortex cores lie almost parallel to the blade chord, the interaction is characterized by a two-stage response associated with the sequential passage of the two cores. Conversely, when the cores lie on a plane that is almost perpendicular to the blade chord, the response is similar to that of a single vortex interaction. In all cases, the normal force response is consistent with the distribution of vertical velocity in the flow field of the vortex system. The pitching moment response, on the other hand, depends on the localized suction associated with the vortex cores as they traverse the blade chord. 


\section{NOMENCLATURE}

c $\quad$ interacting blade chord (m)

$c_{b} \quad$ vortex generator blade chord (m)

Cn sectional normal force coefficient $\left(N / 1 / 2 \rho V_{T I P}^{2} c\right)$

$C m_{1 / 4}$ sectional quarter chord pitching moment coefficient $\left(M / 1 / 2 \rho V_{T I P}^{2} c^{2}\right)$

$C p \quad$ pressure coefficient $\left(P / 1 / 2 \rho V_{T I P}^{2}\right)$

$d \quad$ diameter of twin vortex generator placement circle

$\ell_{v} \quad$ geometric lateral distance of either single generator or centroid of twin generator placement circle from the tunnel centerline (m)

$r \quad$ radial position of measurement array $(\mathrm{m})$

$R \quad$ rotor radius $(\mathrm{m})$

$r_{c} \quad$ vortex core radius $(\mathrm{m})$

$r_{c} * \quad$ Non-dimensional vortex core radius $\left(r_{c} / c_{b}\right)$

$x \quad$ distance along chord (m)

$x_{v} \quad$ perpendicular horizontal distance between the leading edge pressure transducer and vortex generator $(\mathrm{m})$

$y_{v}$ geometric height of either single generator or centroid of twin generator placement circle above or below rotor disk (m)

$U \quad$ free stream velocity $(\mathrm{m} / \mathrm{s})$

$V_{T I P} \quad$ rotor tip velocity $(\mathrm{m} / \mathrm{s})$

$v \quad$ vertical velocity

$v^{*} \quad$ non-dimensional vertical velocity $(v /(\Omega r))$ 


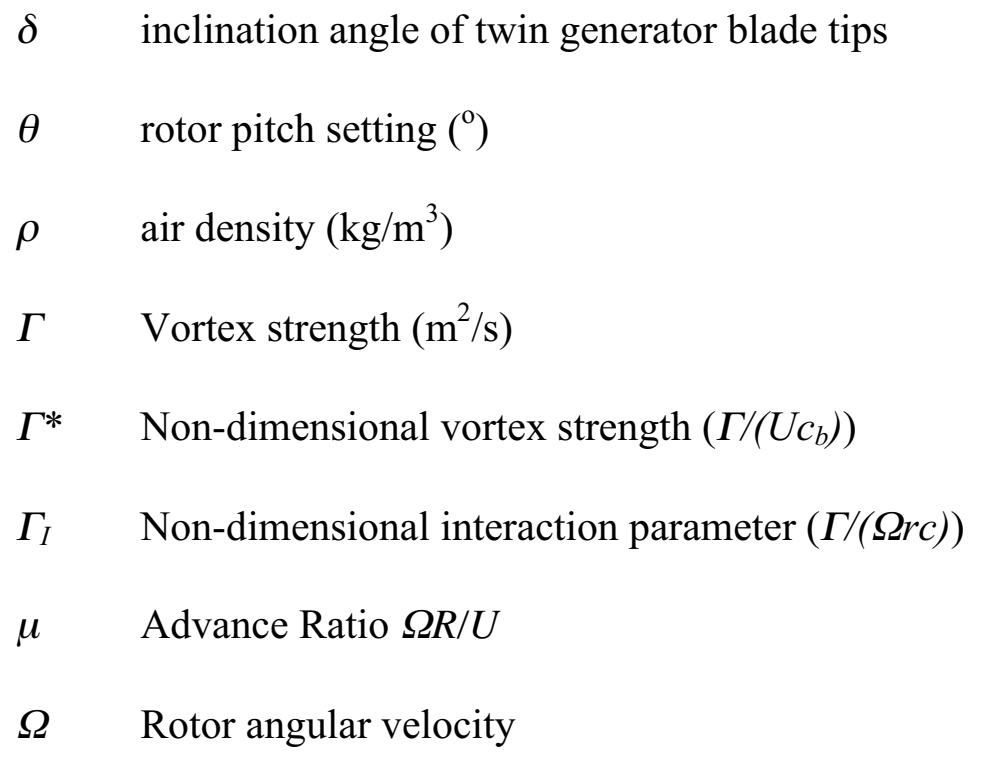




\section{INTRODUCTION}

The tip vortices trailed from helicopter rotor blades may be considered to be the primary features of the highly complex aerodynamic operating environment of the aircraft. In addition to limiting its operational capabilities, these vortices interact with either their originating blade, other blades in the rotor system or the helicopter fuselage to produce unwanted noise and vibration. Much has been done to develop an understanding of vortex generation, convection and interaction including both experimental ${ }^{1-9}$ and numerical studies ${ }^{10-13}$ which have provided a basis for many design improvements. As understanding of the flow phenomena has increased, attention has focused on the potential for both active and passive flow control to ameliorate undesirable aerodynamic effects. Efforts have concentrated on either modifying the rotor tip vortex system by direct intervention during its formation or through changes to the tip planform shape.

The most popular direct intervention method has been the injection of air at the blade tip $^{14}$. Early attempts to do this relied on high levels of blowing to achieve any significant levels of vortex control. More recent studies, however, using discrete jets have demonstrated that significant changes can be made to the vortex structure with relatively

low levels of blowing. For example, Gowanlock et al. ${ }^{15}$, have recently shown that selective use of discrete jets oriented in the spanwise direction, can reduce the peak rotational velocities in the tip vortex by as much as $50 \%$ on a model rotor, even at momentum blowing coefficients as low as 0.0033 . Similarly, Liu et al. ${ }^{16}$ computed the benefits of blowing air at 5\% of the freestream speed from a spanwise slot positioned 
near the tip trailing edge on the upper surface of a rotor blade. They postulated that pulsed blowing from this type of slot could be useful in reducing the higher harmonic loads and noise during a blade vortex interaction (BVI). More recently, Coton et al. ${ }^{17}$ demonstrated that multi-directional blowing at the tip trailing edge can produce an expansion of the vortex core and a corresponding reduction in the severity of near-core BVI events.

Modification of the blade tip planform is not a new concept, in fact tip shapes designed to enhance specific aspects of the helicopter performance are now commonplace on inservice helicopters. Possibly the most notable of these is the Westland Helicopters BERP tip. It has been documented by Scott et. al. ${ }^{18}$ that this tip can generate two trailed vortices at moderate-to-high incidence with the outboard vortex being stronger than the inboard which is of opposite sense. Lowson ${ }^{19}$ also stated that this tip reduces the noise in low speed descent, where BVI is predominant.

It has also been possible to consider the design of tip planforms specifically to modify the tip vortex structure in a manner which potentially reduces the severity of the associated BVI events. Hoad ${ }^{20}$ studied the effects of a variety of different tip planforms on the impulsive noise from blade vortex interaction. Some of these planforms resulted in the generation of a small secondary vortex in addition to the main tip vortex. Others, such as the sub-wing ${ }^{21}$ and later the 'Vane-Tip ${ }^{\prime 2}$ (Fig. 1), distributed the vorticity at the blade tip as a pair of vortices. It has been shown that this type of design has the potential to 
considerably reduce the severity of individual BVI events thus producing a corresponding reduction in noise ${ }^{23}$.

Until relatively recently, little was known about the temporal and spatial development of two such closely spaced trailed vortices and so it is difficult to assess the actual effect that such a tip design would have. A study was conducted at the University of Glasgow to investigate possible methods of generating such a vortex pair in the controlled environment of a wind tunnel and to further investigate the temporal and spatial development of the vortex system ${ }^{24}$. The primary objective of this study was to provide a suitable twin vortex generation method for subsequent blade vortex interaction studies. It was demonstrated that a stable vortex pair could be generated and that the rotation of the pair could be represented in time by a relatively simple two-dimensional induced velocity model. It was also shown that the spacing of the vortex pair and their strengths remained almost constant with downstream distance from the vortex generator. In contrast, the vortex cores grew almost linearly with downstream distance and eventually merged far downstream when the ratio of the separation distance to the vortex core diameter was approximately 1.9 or less as originally suggested by Rossow ${ }^{25}$.

In this paper, a twin vortex system is generated in a low speed wind tunnel and used to study its parallel interaction with a rotating blade set at zero incidence. The results are compared with those from interaction experiments in the same facility involving a single $\operatorname{vortex}^{26}$. 


\section{EXPERIMENT}

\section{Model and test Set-up}

All tests were performed in the University of Glasgow $1.61 \mathrm{~m} \times 2.13 \mathrm{~m}$ low speed, atmospheric pressure, closed return "Handley Page" wind tunnel at a flow velocity of $47 \mathrm{~m} / \mathrm{s}$. Figure 2 illustrates the basic test set-up which consisted of a vortex generator located upstream of a rigid, untwisted single rotor blade. This arrangement is similar to that used by Horner et al. ${ }^{8,9}$.

Two vortex generators were manufactured to produce either a single vortex or twin vortex system. Each vortex generator consisted of two NACA 0015 section cantilever wings of chord $0.1524 \mathrm{~m}$ made of suitably shaped aluminum spars enclosed in glass fiber skins. These wings were positioned at the roof and floor of the mouth of the working section and set at equal and opposite incidences to produce vortices of the required strength and rotational sense. For the single BVI investigations, the generator wing pair was pinned at the blade intersection and was of sufficient length to pass through the tunnel roof and floor. Due to the proximity of the pinned intersection, a single vortex was formed. For the twin vortex generator, the wing tips could be separated laterally and vertically resulting in two distinct rolled up vortices. The resulting vortex system was then convected downstream by the tunnel flow to interact with the clockwise-rotating rotor (viewed from above). This approach to twin vortex generation was adopted on the basis of the previous proof of concept study by Copland et al. ${ }^{24}$. Vertical positioning of the twin vortex generator was achieved by a telescopic arrangement in which the bases of 
the generator wing pair were moved in and out of streamlined fairings. Lateral positioning of the wings was achieved manually.

Velocity measurements of the vortices produced by the two generators were made using a TSI IFA-300 Hot Wire Anemometer system using DANTEC 55P61 cross wire probes. The probes used $5 \mu \mathrm{m}$ diameter platinum plated tungsten wires with a length-to-diameter ratio of 250 . The measuring volume of the probe was approximately $0.8 \mathrm{~mm}$ in diameter and $0.5 \mathrm{~mm}$ in height. The probe was calibrated in a special wind tunnel dedicated to the purpose. During calibration, the probe was rotated (in $6^{\circ}$ steps) $\pm 30^{\circ}$ in the plane of the sensor wires to determine yaw sensitivity. A full account of the calibration procedures used can be found in Copland ${ }^{27}$.

The interacting rotor blade, of NACA 0015 cross-section, was manufactured from carbon fiber using a purpose built Nylatron mould and had a chord of $0.1524 \mathrm{~m}$ and a span of $0.944 \mathrm{~m}$ (based on rotor disk radius) resulting in an aspect ratio of 6.19. The blade merged to a tapered circular section at the root which was located within an aluminum hub and counterweight assembly. The tapered section provided the centrifugal pull-out load reaction force, whilst the circular section and pitch grip clamp allowed the rotor pitch angle to be set at discrete values. The blade was instrumented with 72 miniature Kulite pressure transducers located at the spanwise locations shown in Table 1 and on the dashed lines shown in Figure 3. This allowed detailed chordwise and spanwise pressure data to be collected along the blade during a BVI event. 
The rotor blade, hub and counterweight were mounted on a hollow cylindrical drive shaft located vertically in the test section and passing through the tunnel roof and floor (Figure 1). The rotor was driven at $600 \mathrm{rpm}(10 \mathrm{~Hz})$ by a $\mathrm{DC}$ electric motor and bevel gear arrangement located above the tunnel roof. The resultant rotor speed provided a tip Mach number of 0.17 and a nominal tip Reynolds number of 600,000 . The electrical wiring from the pressure transducers was passed through the drive shaft, and the signals were collected via a slip ring set by a BE256 data logger. All data were recorded over a $144^{\circ}$ capture angle at $50 \mathrm{kHz}$ sampling rate with 32,000 samples being logged per channel, per run, over 16 revolutions. The resulting azimuth angle resolution between samples was $0.072^{\circ}$.

The flow velocity and rotor rotational speeds used in the experiment correspond to an advance ratio $\mu=0.79$ which contrasts with the nominal range of $0.15-0.2$ generally associated with BVI on a helicopter rotor. One consequence of this is that unrepresentative local flow skew angles exist in the measurement region of the blade and will inevitably influence the measured data. Comparisons of previous measurements in the same facility with other experiments and numerical predictions ${ }^{8}$, however, suggest that the collected data will exhibit the essential features of parallel BVI.

\section{Test Geometry}

The test geometry used for the single BVI investigations is illustrated in Figure 4. All parameters were taken relative to the vortex generator wing junction located 2.1 rotor 
radii upstream of the rotor. Parameter $\ell_{v}$ was used as a measure of the lateral offset of this junction from the tunnel centerline which, for parallel interactions, was zero.

In order to provide a temporal reference frame for a BVI interaction, the $x_{v}$ parameter was used. This provided a measure of the perpendicular distance between the generator wing junction and the leading edge pressure transducer at a particular station. Hence, $x_{v}$ was a function of both blade station, $r / R$, and blade azimuth angle.

Figure 5 illustrates the test geometry used for the twin BVI investigations. For this case, parameters $x_{v}$, and $\ell_{v}$ were taken relative to the centre of the placement circle about which the top and bottom wings of the twin vortex generator were positioned. This placement circle also introduced a slight obliqueness within the twin BVI events considered. Additional parameters were used to geometrically position the twin generator wings on the circumference of this circle, namely the placement circle diameter, $d$, and the blade angular position on the placement circle, $\delta$.

It should be noted that all the above parameters are geometrical in origin and do not account for the effects of vortex meander.

The twin vortex test program was performed for a rotor pitch setting of $\theta=0^{\circ}$ only. The centre of the generator placement circle was placed in the $\ell_{v} / c=0$ position and at heights of $y_{v}=0, \pm 0.2 \mathrm{c}$, about which points the placement circle diameter, $d$, was set at either 
$0.5 \mathrm{c}, 0.75 \mathrm{c}$, or $1 \mathrm{c}$. The angular position of the twin vortex generator, $\delta$, together with the other parameter settings used in the test program are detailed in Table 2.

In the following discussion, BVI measurements corresponding to cases in which there was no lateral or vertical offset of the vortex system, i.e. $y_{v}=\ell_{v}=0$, are presented. Twin vortex BVI measurements are presented for a range of vortex generator angular positions but with the core separation and individual vortex strength fixed at $0.5 \mathrm{c}$ and $2.9 \mathrm{~m}^{2} / \mathrm{s}$ respectively. These results are compared with a single vortex parallel BVI interaction case with a vortex strength of $5.8 \mathrm{~m}^{2} / \mathrm{s}$, corresponding to a generator blade incidence or $12.5^{\circ}$. It should be noted that this is the same incidence setting as that used on the twin vortex generator to generate the weaker twin vortices.

\section{Experimental Errors}

The data acquisition system and software has an automatic gain adjustment feature that allows measurements to be taken at the maximum resolution of the system. On the basis of previous experience with Kulite miniature transducers ${ }^{26}$ and taking account of discretization and calibration factor errors, the uncertainty in the measured pressure coefficients is estimated to be $0.5 \%$.

The blade pitch of the vortex generator could be set to an accuracy of better than $0.25^{\circ}$. The vortex strengths, determined from the hot-wire measurements, were accurate to within $2 \%$ and the corresponding estimates of core size to within 5\%. A significant source of uncertainty in the measurements was the position of the vortex system. Once produced by the rotor rig, the vortex system convected through the wind tunnel and was 
subject to forcing from the rotating blade in the working section. Although the position of the vortex generator could be set to within $0.5 \mathrm{~mm}$, the vortex system was found to meander. The maximum meander, has been estimated using PIV to be around $40 \mathrm{~mm}$; the primary effect is a change in vortex height. To provide an indication of the effect this has on the normal force and pitching moments, plots of several cycles of data are presented in the results section.

\section{RESULTS AND DISCUSSION}

\section{Vortex Characteristics}

In the present study, measurements of both the twin and single vortex systems were made with the hot-wire anemometry system. These measurements were made using an X-wire probe in the BVI measurement plane but in the absence of the rotor rig and were used to establish the relationship between the vortex generator settings and the resulting vortex systems. The technique used to determine the individual strengths of each of the twin vortices followed that used in a previous study in a smaller wind tunnel by Copland et $\mathrm{al}^{24}$. In essence, the cross-flow velocities due to the twin vortex system were measured on a two-dimensional grid at a specific location downstream of the vortex generator. The vortex core locations were then input to a numerical model based on superposition and the core sizes and vortex strengths adjusted until a best fit was obtained between the 
model and the experimentally measured flow field. Full details of this method and example comparisons between the model and experimental data are provided in Ref. 24 .

The variation of vortex strength is plotted against the incidence setting of the generator wings for both the twin and single vortex cases in Fig. 6. From this figure, it may be observed that, for a given generator wing incidence, the strength of the single vortex is twice that of each of the twin vortices. In essence, this implies that in the former case the vorticity from the two generating wings is channeled into one intersection vortex, whereas, in the latter, the vorticity from each wing is channeled into its own isolated tip vortex.

In their previous study, Copland et al. ${ }^{24}$ found that, the core sizes of the vortices produced by the generator were insensitive to the wing incidence. This is confirmed in the present case by Fig. 7 which shows that the variations in the core sizes of both the twin and single vortices with wing incidence are very small. Interestingly, however, the core size of the single vortex is around $30 \%$ larger than those of the twin vortices.

Generally, it was found that the measurements of vortex core size and strength were repeatable for a given generator wing incidence, regardless of the wing tip location in the generator placement circle. Copland et al. ${ }^{24}$ noted, in the previous study, that the spacing between the two vortex cores showed some dependency on the orientation of the vortices with respect to each other. This effect was attributed to interference from the probe traversing system and was again observed in the present study. This effect introduces 
some uncertainty in the measured orientation and spacing of the vortices. Table 3 shows the measured geometric characteristics of three different twin vortex configurations in the BVI measurement plane. The three vortex systems were produced with a spacing of $0.5 \mathrm{c}$ between the vortex generator wings and $\delta$ settings of $0^{\circ}, 45^{\circ}$ and $90^{\circ}$. Although the measurements exhibit variability in core spacing, it should be noted that this should not be attributed solely to traverse interference since the roll-up process is also influential. It is known that tip vortices roll up slightly inboard of the wing tip which, in the $\delta=90^{\circ}$ case where the wing tips were separated vertically, would result in an increased spacing between the cores in line with the measured data. Possibly, however, the most interesting feature of the measurements is the angular difference between the three vortex systems. In terms of the tips of the vortex generator wings, the three cases are progressively spaced by $45^{\circ}$ whereas the measurements indicate a progressive rotation of more than $75^{\circ}$ between the vortex systems. The reason for this is unclear but the way in which the roll up process is influenced by the mutual inductance between the two wings may be a factor in this. The previously mentioned traverse interference is also a possible contributory factor although, from the BVI results presented in the following section, it appears that the measured orientations are not significantly different from those experienced by the interacting blade in the absence of the traverse.

\section{BVI Measurements}

In this section all aerodynamic data were non-dimensionalized using the rotor tip velocity, $V_{T I P}$. This resulted in comparable aerodynamic scaling between the rotor and vortex generator. To aid interpretation of the twin vortex BVI results, a case will be 
presented in which the blade interacts with a single vortex. This case is typical of measurements that have been fully described elsewhere ${ }^{26}$, but its description here will allow the gross features of the basic interaction to be characterized before consideration of the more complex twin case.

\section{Single Vortex Interaction}

Figure 8 illustrates the temporal variation of the upper surface pressure distribution during a parallel interaction $\left(\ell_{v} / c=0, y_{v} / c=0\right)$ with a vortex of strength $\Gamma=5.8 \mathrm{~m}^{2} / \mathrm{s}$ at the inboard $(r / R=0.785)$ station. It should be noted that the data, in this figure, are from one of the sixteen recorded revolutions for this particular case. The plot shows $-C p$ on the vertical axis plotted against the transducer chord position, $\mathrm{x} / \mathrm{c}$, and non-dimensional time, $x_{v} / c$, in the foreground. It should be noted, at this stage, that the vortex was prone to meandering during the experiment. This can be attributed to the forcing of the rotating blade in the wind tunnel working section since the vortex was stable during the hot-wire measurements described above. This meandering effect has been observed during previous BVI experiments at Glasgow ${ }^{7,8,9}$ and its magnitude has been estimated, using Particle Image Velocimetry, to be around $0.25 \mathrm{c}$. Thus, given the potential variability between individual revolutions, it was inappropriate to cycle average the data as this would have resulted in attenuation of significant local features of the response. Great care has, therefore, been taken to ensure that the individual interactions presented in this paper are representative of the general behavior exhibited in the sixteen revolutions collected for each case. 
The plot presented in Fig. 8 illustrates the typical upper surface response to blade vortex interaction, observed in previous studies, for a rotor pitch of $0^{07,8,9}$. It shows the characteristic slow increase in leading edge suction peak associated with the flow field induced by the approaching vortex. This is followed by a rapid collapse of this peak as the vortex moves onto and over the leading portions of the blade in the $0<x_{v} / c<0.35$ region. After this, a more clearly defined low-pressure ridge associated with the vortex convection over the blade is seen until the vortex finally leaves the blade at approximately $x_{v} / c=0.92$. Following this, the trailing edge region remains disturbed for a short time before gradually returning to its undisturbed state. The origin of this disturbance was examined in PIV studies at Glasgow'. These showed that subsequent to the passage of the vortex off the trailing edge, the rear most portions of the blade remain in a highly disturbed flow field as the vortex initially moves downwards and then upwards in the blade wake.

The behavior on the lower surface of the blade during such an interaction has been observed in previous studies and will, for completeness be described briefly here. Due to the sense of the vortex, the flow velocities on the lower surface of the blade are inhibited and no large suction region occurs as the vortex approaches the blade. Rather, the pressures aft of the stagnation point on the forward portion of the lower surface increase slightly as the vortex approaches the blade. Once the vortex passes the leading edge, there is a recovery in the lower surface suction pressures as the low pressures associated with the impinging vortex begin to dominate. The low-pressure ridge associated with the passage of the vortex across the surface is also observed although the vortex takes longer 
to pass over the lower surface of the blade than the upper. This may be due to influence of the blade bound circulation slowing its progression.

Figure 9 illustrates the sectional normal force coefficient non-dimensional time histories obtained by integrating the upper and lower surface pressures at the 0.785 span station for the case presented in Fig. 8. It may be observed that $\mathrm{Cn}$ steadily increases as the vortex approaches the blade and reaches a maximum value just after $x_{v} / c=0$. Following this, the normal force decreases abruptly, flattens out and then following a sudden decrease in $\mathrm{Cn}$ near $x_{v} / c=1$, gradually tends towards zero. Consideration of this plot together with Fig. 8 allows the $\mathrm{Cn}$ response to be explained in terms of the $C p$ data. In particular, the initial rise followed by the sudden reversal in $C n$ occurs as the vortex first approaches and then convects over the front section of the blade. The resulting suction peak produces the corresponding maximum $C n$ value just after the $x_{v} / c=0$. Following this, the collapse in suction pressures on the forward portion of the blade results in the rapid reduction in $\mathrm{Cn}$. Subsequently, the relatively constant upward force increment associated with the low pressure vortex convection ridge, halts the drop in the normal force. This causes the $\mathrm{Cn}$ values to level off until the vortex convects off the trailing edge of the blade. The following negative spike corresponds to the loss of vortex induced upward force on the rotor as the low pressure vortex convects off the blade. In addition, the vortex flow field also causes a corresponding increase in the local pressures recorded at the trailing edge of the lower surface which further contributes to the magnitude of this spike. This induced effect then decreases as the vortex convects away from the blade, and the $\mathrm{Cn}$ tends towards zero. 
Figure 10 presents the temporal variation in the quarter chord sectional pitching moment coefficient obtained by integration of the chordal pressure distribution at the 0.785 span station for the same case as before. It may be observed that, as the vortex approaches the leading edge, there is a very gradual increase in $C m_{1 / 4}$. Shortly after the peak $C n$ is attained, the $\mathrm{Cm}_{1 / 4}$ reaches a maximum and then steadily decreases until becoming almost neutral. Finally, as the vortex passes the trailing edge, there is a sharp upward spike in the $\mathrm{Cm}_{1 / 4}$ curve before returning to a negative value which slowly tends towards zero.

The behavior exhibited in the $\mathrm{Cm}_{1 / 4}$ curve can, once again, be explained in terms of the pressure response. As indicated above, the approach of the vortex causes an increase in the nose up moment which continues past the $x_{v} / c=0$ region. This delay in recording the maximum $\mathrm{Cm}$ value is thought to be due to a greater net suction occurring on the forward portion of the blade as the low pressure vortex first convects onto the rotor. The $\mathrm{Cm}_{1 / 4}$ values then drop as the vortex convects past the quarter chord. This effect is produced by the low pressure vortex convection ridge which generates an increasing negative nose down pitching moment, as its moment arm increases during its movement towards the trailing edge. This continues until the vortex convects off the blade where the sudden gain in negative $C n$ at the trailing edge and the large moment arm combine to produce a fast acting surge in $\mathrm{Cm}_{1 / 4}$. This then reverses as the vortex moves away from the blade. As the blade continues to move, this influence is further reduced and the pitching moment slowly returns to a zero value. 


\section{Twin Vortex Interaction}

In the following discussion, data are presented for twin BVI events involving vortex pairs with an initial separation, $d / c$, of $0.5 c$. Cases are presented for a range of vortex generator inclination angles to examine how the nature of the interaction changes with the orientation of the vortex system. In all cases, the parameters $y_{v} / c$ and $\ell_{v} / c$ were set equal to zero and each vortex had a strength of $\Gamma=2.9 \mathrm{~m}^{2} / \mathrm{s}$ giving a combined vortex strength of the twin system of $\Gamma=5.8 \mathrm{~m}^{2} / \mathrm{s}$.

Figure 11 presents the temporal variation in the upper surface pressure distribution measured during a parallel BVI with the vortex system identified as Case 1 in Table 3. As mentioned previously, 16 revolutions of data were recorded for each interaction case and Fig. 11. presents interaction data from three of the revolutions recorded for this case. These three data sets are representative of the range of responses measured for this particular case. In Fig 11a, cycle 1, the upper surface response exhibits some of the features observed in the single vortex interaction. In particular, the characteristic slow increase in leading edge suction peak associated with the flow field induced by the approaching vortex system is again evident. In this case, however, the rapid collapse of the leading-edge suction peak is followed by the formation and subsequent collapse of a weaker second peak. The response over the remainder of the chord is dominated by two low pressure ridges emanating from the leading edge peaks. These ridges, indicative of the passage of the twin vortex cores, appear to maintain almost uniform spacing during their passage down the chord. After the first vortex leaves the trailing edge, the trailing 
edge pressure response becomes disturbed and this continues up to and beyond the passage of the second vortex.

A quite different response is obtained in Fig. 11b, cycle 10, where, although the pressure levels before and after the interaction are almost identical to cycle 1 , the interaction itself is quite different. Here, the severity of the leading edge response is greatly reduced and the second suction peak is barely discernible. There is also little evidence of the two suction ridges associated with the passage of the vortex system over the blade.

The response exhibited in Fig. 11c, cycle 13, is almost identical to that in cycle 1. The only obvious differences in the two plots are a higher secondary leading-edge peak in cycle 13 and the reduced disturbance at the trailing edge subsequent to the passage of the vortex system. Otherwise, both plots display the same general features.

A fuller understanding of the interaction characteristics can be gained by examination of the corresponding lower surface pressure responses for the three cases presented above. The lower surface response for cycle 1 is shown in Fig. 12a. As the vortex reaches the blade leading edge, there is a significant increase in suction at the leading-edge transducer. Aft of the stagnation point, however, the local pressure can be observed to increase in a manner that is consistent with the single vortex interaction case described earlier. Once the vortex passes the leading edge, there is a noticeable increase in suction across the entire chord. Interestingly, however, there is no obvious indication of the passage of the vortex across the chord. 
Figure $12 \mathrm{~b}$ presents the lower surface response corresponding to cycle 10 . This case exhibits the same gross features as cycle 1 but the detail response is quite different. In particular, two clear ridges associated with the chordwise convection of the vortex system are evident in this case.

Finally, Fig 12c presents the lower surface response measured during cycle 13 . The early stages of the response in Fig. 12c are almost identical to cycle 1 but the latter stages are quite different. Both figures show a two stage rise in suction as the vortex system moves over the blade but the second stage response in Fig. 12c is much more dramatic than in Fig. 12a.

The behavior exhibited in Figures 11 and 12 is illustrative of the extent to which vortex meander can significantly change the detail of the interaction. For this case, Table 3 suggests that both vortices in the system lie in the plane of the blade. Thus, on a purely geometric basis, it would be expected that the blade would cut the vortices one after the other. If this were to happen, it would also be expected that the passage of the vortex fragments over the upper and lower surfaces would be marked by the appearance of convective ridges of the type observed in Fig. 11a and Fig 12b. This is clearly not happening as, in each of the cycles presented, the ridges only appear on one surface. In other words the vortex system is being convected either above (cycles 1 and 13) or below (cycle 10) the blade. This is not unexpected as previous studies in the same facility have shown that the vortex system is prone to meandering in this way with the vortex core 
tending to pass either above or below the blade rather than impacting directly on the leading edge of the blade. This is likely to be an artifact of the experimental configuration, as it has been shown that vortex meander can be avoided in carefully designed tests ${ }^{28}$, although vortex meander will occur during helicopter flight due to, for example, atmospheric turbulence.

Although cycles 1 and 13 are very similar, the slight differences observed may be attributable to another aspect of the behavior of the vortex system. The almost identical response in the two cycles as the first vortex approaches the blade suggests that the position of the first vortex with respect to the plane of the blade is the same in the two cases. The rise in suction due to the approach of the second vortex is, however, much weaker in cycle 1. This suggests that the second vortex is further away from the plane of the blade in cycle 1. This may be due either to inclination of the vortex system with respect to the plane of the blade or convection of the entire system away from the blade as the interaction progresses. Since the vortex system was found to be spatially stable during the hot-wire measurements, it is likely that the forcing from the rotating blade is, again, responsible for this variability from cycle to cycle.

Similar cycle-to-cycle differences exist for all three cases documented in Table 3. Figure 13 again illustrates these variations, by presenting integrated normal force data for eight consecutive cycles, but also compares the gross response for each of the three vortex orientations. Fig. 13a presents the integrated normal force coefficients for Case 1 in Table 3. Despite the variability, the gross response retains many of the features of the 
single vortex interaction. In particular, the normal force rises during the early stages of the interaction before falling sharply as the vortex system crosses the leading edge of the blade before dropping to a minimum value from which there is a progressive but slow recovery. The magnitude of the change in normal force is also only marginally less than the single vortex interaction. Some features do, however, distinguish the response from that of the single vortex. In particular, the overall duration of the interaction is increased as a consequence of the spacing between the twin vortex cores. In addition, the downward trend in normal force, after the initial interaction, is arrested, and even reversed, for a short time as the upwash from the second of the two vortices produces a recovery in the leading edge suction peak. Following this, the rate of decrease in normal force is greater than during the initial stages of the interaction possibly as a consequence of either translation or rotation of the vortex system during the interaction.

Fig. $13 \mathrm{~b}$ presents the corresponding normal force data for Case 2 where the inclination angle of the vortex pair is expected to be around 75 degrees. The degree of scatter in the data is similar to Case 1 but the characteristics of the interaction are noticeably different. Firstly, the magnitude of the change in normal force during the interaction is considerably smaller than the previous case. In addition, the drop in normal force after the initial interaction is almost monotonic with little evidence of the influence of a 'second' vortex.

In the final case, Fig 13c, the normal force response is similar to Case 1 with the recovery in normal force associated with the passage of the second vortex clearly visible. The overall magnitude of the response is, however, marginally less than Case 1 and it also 
appears to have a longer duration. These features are consistent with the increased separation distance between the two vortices in the measurement plane, identified by the hot-wire measurements.

As mentioned previously, Copland et al. ${ }^{24}$ showed that the twin vortex system could be modeled in a two-dimensional plane by a relatively simple superposition technique. Using this technique and based on the measured vortex parameters it is possible to reconstruct the velocity field that the blade travels through for each of the twin vortex inclination angles. A major factor in determining the BVI response is the vertical velocity experienced by the blade during the interaction. In fact, this is often used as a basis for semi-empirical BVI calculations ${ }^{29}$. The vertical velocity components have been extracted from the numerical model and are presented in Fig. 14 for the three cases discussed above. In each case the vortex inclination angle and separation distance in the measurement plane have been supplied as input to the numerical model. In addition, the corresponding vertical velocity distribution produced by a single vortex of the combined strength of the twin vortices is also shown.

It is immediately obvious that the basic form of the normal force response for each of the cases is mirrored by the corresponding vertical velocity distribution. In particular, the vertical velocity distributions for Cases 1 and 3 both show the characteristic double peak exhibited by the interaction data. Similarly, the vertical velocity distribution for Case 2 has a similar form to the single vortex velocity field, albeit somewhat reduced in magnitude, and this is consistent with the normal force response in the two cases. The 
increased extent of the velocity field and the decrease in peak vertical velocity associated with the increased spacing between the vortices in CASE 3 is also a notable feature of the plot.

Further insight into the nature of the twin vortex interaction can be gained by examination of the blade quarter chord pitching moment response during the interaction. Of the cases presented above, Case 1, where the vortex cores are parallel to the chord line produces the most pronounced two-stage response in the normal force coefficient time history. The corresponding pitching moment data are presented for this case in Fig. 15. It is immediately obvious that these data exhibit considerably more noise than the corresponding normal force data. This arises because of the increased sensitivity of the integrated pitching moment coefficient to the small number of measurement points around the profile of the blade. Nevertheless, the figure does highlight the basic features of the pitching moment response for this case. The same gross features identified in Fig. 10 , for the single vortex interaction, are also present in this figure. The progressive rise in $\mathrm{Cm}_{1 / 4}$ prior to the interaction and the subsequent drop towards a neutral value are common to both figures. The strong upwards spike in the pitching moment identified with the passage of the vortex over the trailing edge in Fig. 10 was a key feature of the single vortex distribution. In the twin vortex case, many of the individual traces exhibit two spikes, presumably corresponding to the sequential passage of the two vortices over the trailing edge. This result is consistent with the observations of Horner et al. ${ }^{9}$ in their study of single vortex interactions who suggested that, while the normal force response is dominated by the global velocity field associated with the vortex system, the pitching 
moment response is dominated by the local suction produced by the proximity of the vortex core to the surface of the blade.

\section{CONCLUDING REMARKS}

Details are given of a Blade Vortex Interaction (BVI) test series performed in the University of Glasgow $1.61 \mathrm{~m}$ x 2.13m low speed, closed return, 'Handley Page' wind tunnel, in which single and twin vortex interactions with a rigid, unloaded model rotor blade were investigated.

A specially designed vortex generator allowed the rotational position of the twin vortex pair to be varied so that a range of interaction geometries could be studied. Hot wire anemometry was used to measure strength, size and orientation of the vortex pairs in the interaction plane.

It was found that blade surface pressures, normal force and pitching moment responses strongly depended on the orientation of the vortex pair in the interaction plane. When the vortex cores lay almost parallel to the blade chord, the interaction was characterized by a two-stage response associated with the sequential passage of the two cores. Conversely, when the cores lay on a plane that was almost perpendicular to the blade chord, the response was similar to that of a single vortex interaction. 
It was shown that, for all cases, the normal force response was consistent with the expected distribution of vertical velocity in the flow field. It was also demonstrated that while the normal force response was dominated by the global velocity field, the pitching moment response depended on the localized suction associated with the vortex cores.

It is important to note that the flow velocity and rotor rotational speeds used in the current study correspond to a much higher advance ratio than is generally associated with BVI on a helicopter rotor. This will inevitably result in differences between the fluid dynamic response measured here and that which may be expected in flight. Previous experience suggests, however, that these differences are likely to be relatively minor. In addition, although the present results highlight the general features of the blade response to a twin BVI, there are numerous sources of unsteadiness in the experiments that introduce variability from cycle to cycle of the interacting blade. It is hoped to gain further insight into the detail of the interaction process in the future by simultaneously capturing both surface pressure and global flow field information during individual interactions.

\section{ACKNOWLEDGEMENT}

The authors wish to acknowledge the significant contributions of Mr. Allan Masson and Dr. Christopher Copland to the experimental work described in this paper. 


\section{REFERENCES}

${ }^{1}$ Padakannaya R. "Experimental Study of Rotor Unsteady Airloads due to Blade Vortex Interaction", NASA CR - 1909, November, 1971.

${ }^{2}$ Ham, N.D., "Some Conclusions from an Investigation on Blade-Vortex Interaction", Journal of the American Helicopter Society, Vol. 20, pp 26-31, October, 1975.

${ }^{3}$ Caradonna, F.X., Laub, G.H. and Tung, C. "An Experimental Study of Rotor-Vortex Interaction", NASA TM-86005, November, 1984.

${ }^{4}$ Booth, E.R.Jr., and Yu, J.C., "Two-Dimensional Blade-Vortex Flow Visualisation Investigation", AIAA Journal ,Vol. 24, No. 9, September, 1986.

${ }^{5}$ Caradonna, F.X., Lautenschlager, J.L. and Silva, M.J., "An Experimental Study of Rotor-Vortex Interactions", AIAA Paper 88-0045, AIAA 26th Aerospace Sciences Meeting, Reno, Nevada, January, 1988.

${ }^{6}$ Straus, J., Renzoni, O. and Mayle, R.E., "Airfoil Pressure Measurements During a Blade-Vortex Interaction and a Comparison with Theory", AIAA Paper 88 0669, AIAA 26th Aerospace Sciences Meeting, Reno, Nevada, January, 1988. 
${ }^{7}$ Horner, M.B., Saliveros. E., and Galbraith, R.A.McD. "An Examination of Vortex Convection Effects During Blade-Vortex Interaction, The Aeronautical Journal, Vol. 96, Number 960, December 1992.

${ }^{8}$ Horner, M.B., Saliveros, E., Kokkalis, A., and Galbraith, R.A.McD. "Results from a Set of Low Speed Blade-Vortex Interaction Experiments", Journal of Experiments in Fluids, 14, pp. 341-352, May 1993.

${ }^{9}$ Horner, M.B., Stewart, J.N., Galbraith, R.A.McD., Grant, I., Coton, F.N., “An examination of vortex deformation during blade-vortex interaction utilising particle image velocimetry", AIAA Journal, Vol. 34, No. 6, pp. 1188-1194, 1996

${ }^{10}$ Sears, W.R., "Aerodynamics, Noise and the Sonic Boom", AIAA Journal, Vol. 7, No. 4, April, 1969.

${ }^{11}$ Panaras, A., "Numerical Modelling of the Vortex- Airfoil Interaction", AIAA Journal, Vol. 2S, No. 1, January, 1987.

${ }^{12}$ Lee, D.J. and Smith, C.A., Distortion of Vortex Core during Blade/Vortex Interaction", AIAA Paper 87- 1243, AIM 19th. Fluid Dynamics, Plasma Dynamics : Lasers Conference Honolulu, Hawaii, June, 1987. 
${ }^{13}$ Jones, H.E., and Caradonna, F.X., "Full Potential Modelling of Blade-Vortex

Interactions", Vertica, Vol. 12 No. 1/2, pp. 129-145, 1988.

${ }^{14} \mathrm{Wu}$, J.M., Vakilli, A., Chen, Z.L., "Investigation of the effects of discrete wingtip jets", $21^{\text {st }}$ AIAA Applied Sciences Conference, AIAA paper no. 83-0546, Reno, Jan 1983

${ }^{15}$ Gowanlock, D.K. and Matthewson, C.S., "Control of rotor tip vortices", $37^{\text {th }}$ AIAA Applied Sciences Conference, AIAA paper No. 99-0012, Reno, Jan 1999

${ }^{16}$ Liu, Z., Sankar, L.N., Hassan, A.A., “Alteration of the tip vortex structure of a hovering rotor by blowing", 37 AIAA Applied Sciences Conference, AIAA paper No. 99-0906, Reno, Jan 1999

${ }^{17}$ Coton, F.N., Green, R.B., Early, J.M., Wang, T., “An investigation of the effect of tip vortex mass injection on blade-vortex interaction", Proceedings of the 27th European Rotorcraft Forum, Moscow, pp. 58.1-58.7, 2001

${ }^{18}$ Scott M.T., Sigl D., Strawn R.C., "Computational and Experimental Evaluation of Helicopter Rotor Tips for High -Speed Flight”, J.Aircraft Vol. 28, pp. 403-409, June 1991.

${ }^{19}$ Lowson M.V., "Progress Towards Quieter Civil Helicopters”, The Aeronautical Journal, Vol. 96, No. 956, pp 209-223, June/July 1992. 
${ }^{20}$ Hoad, D.R., "Helicopter Model Scale Results of Blade Vortex Interaction Impulsive Noise as Affected by Tip Modification", $36^{\text {th }}$ American Helicopter Society Forum, May 1980

${ }^{21}$ Tangler, J.L., 'Experimental investigation of the subwing tip and its vortex structure', NASA CR-3058, 1978

${ }^{22}$ Brocklehurst A., Pike A.C., "Reduction of B.V.I. Noise Using a Vane Tip", A.H.S. Aeromechanics Specialists Conf., San Francisco, Jan. 1994.

${ }^{23}$ Tyler D.J., Vincent A.H., "Future Rotorcraft Technology Developments", The Aeronautical Journal, Vol. 100, No. 1000, pp 451-460, Dec.1996.

${ }^{24}$ Copland, C.M. , Coton, F.N., Galbraith, R.A.McD., "An experimental study on the idealised vortex system of a novel rotor blade tip", The Aeronautical Journal, Vol. 102, No. 1017, pp.385-392, 1998

${ }^{25}$ Rossow V.J., "Convective Merging of Vortex Cores in Lift-Generated Wakes", $J$. Aircraft, Vol. 14, 283-290, 1977. 
${ }^{26}$ Masson, C.A., Green, R.B., Galbraith, R.A.McD., Coton, F.N., "Experimental investigation of a loaded rotor blade's interaction with a single vortex", The Aeronautical Journal, Vol. 102, No. 1018, pp.451-458, 1998

${ }^{27}$ Copland, C.M., "The Generation of Transverse and Longitudinal Vortices in Low Speed Wind Tunnels", Ph.D. Thesis, The University of Glasgow, 1997

${ }^{28}$ Caradonna, F. X, Henley, E., Silva, M., Huang, S., Komerath, N. M., Reddy, U. C., Mahalingam, R., Funk, R. B., Wong, O., Ames, R., Darden, L.A., Villareal, L., Gregory,J., “An Experimental Study of a Rotor in Axial Flight”, AHS Technical Specialists Meeting for Rotorcraft Aeroacoustics and Aerodynamics, October 1997, Williamsburg,VA.

${ }^{29}$ Beddoes, T.S., “Two and Three Dimensional Indicial Methods for Rotor Dynamic Airloads", AHS National Specialist's Meeting on Rotorcraft Dynamics, Arlington, Texas, Nov. 1989 
Table 1. Pressure transducer locations $(x / c)$ on rotor blade

\begin{tabular}{ccc}
\hline \hline $0.785 r / R, 0.985 r / R$ & $0.825 r / R, 0.865 r / R, 0.905 r / R, 0.945 r / R$ \\
upper & lower & upper \\
\hline 0 & & 0 \\
0.009 & 0.011 & 0.501 \\
0.029 & 0.037 & \\
0.059 & 0.069 & \\
0.099 & 0.118 & \\
0.145 & 0.183 & \\
0.177 & 0.276 & \\
0.224 & 0.387 & \\
0.303 & 0.502 & \\
0.401 & 0.630 & \\
0.501 & 0.778 & \\
0.578 & 0.861 & \\
0.650 & 0.925 & \\
0.714 & & \\
0.775 & & \\
0.834 & & \\
0.895 & & \\
0.948 & & \\
\hline
\end{tabular}


Table 2. Twin vortex interaction test matrix

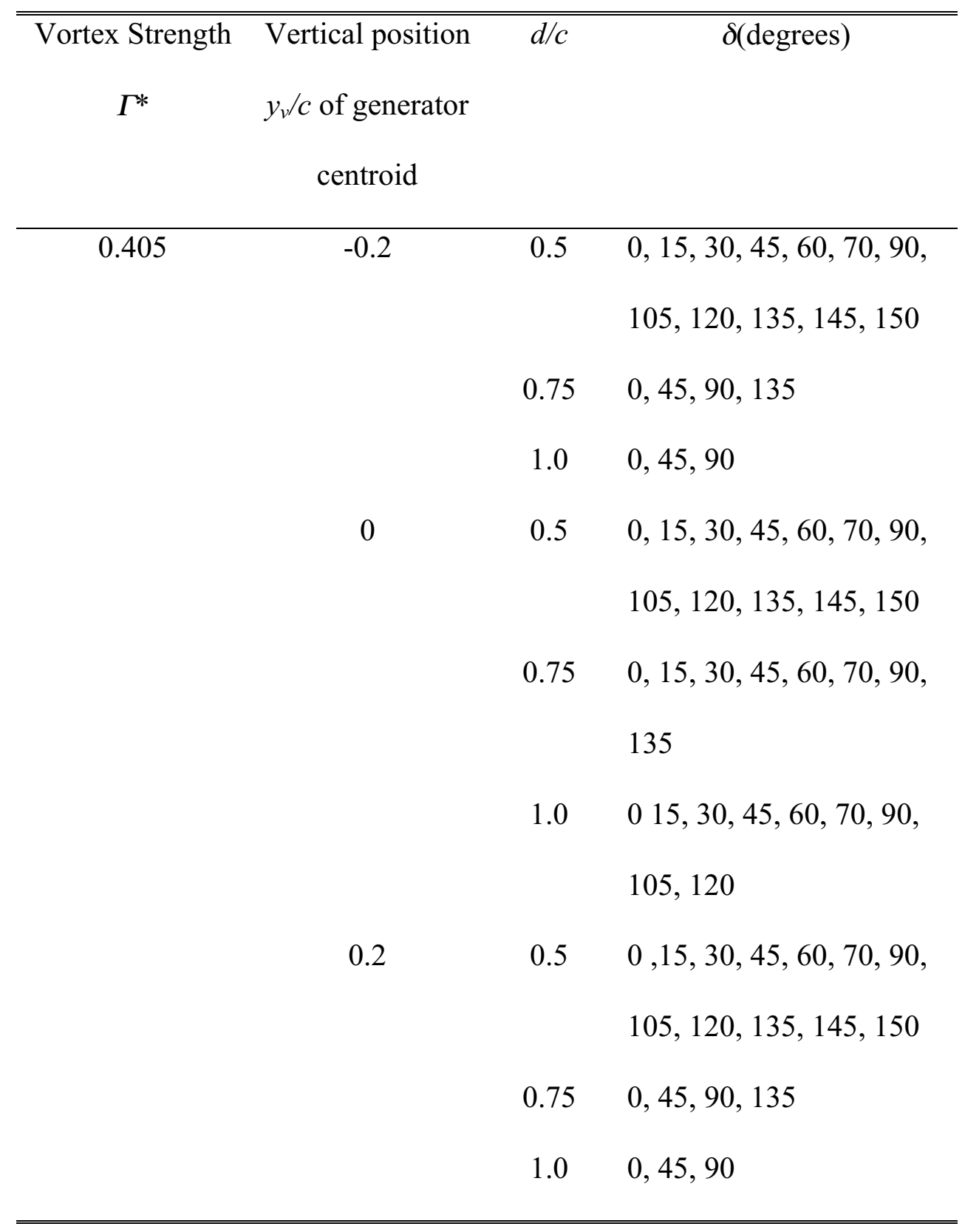


Table 3. Specific twin vortex test cases

\begin{tabular}{cccc}
\hline \hline Case No & $\mathbf{1}$ & $\mathbf{2}$ & $\mathbf{3}$ \\
\hline \multicolumn{4}{c}{ Generator Settings } \\
\hline $\boldsymbol{d} / \boldsymbol{c}$ & 0.5 & 0.5 & 0.5 \\
$\boldsymbol{\delta}$ & $0^{\circ}$ & $45^{\circ}$ & $90^{\circ}$ \\
$\boldsymbol{\theta}$ & $12.5^{\circ}$ & $12.5^{\circ}$ & $12.5^{\circ}$ \\
\hline $\boldsymbol{C}$ & 0.47 & 0.49 & 0.79 \\
$\boldsymbol{d} / \boldsymbol{c}$ & 0.00 & 75.41 & 161.71 \\
\hline \hline
\end{tabular}




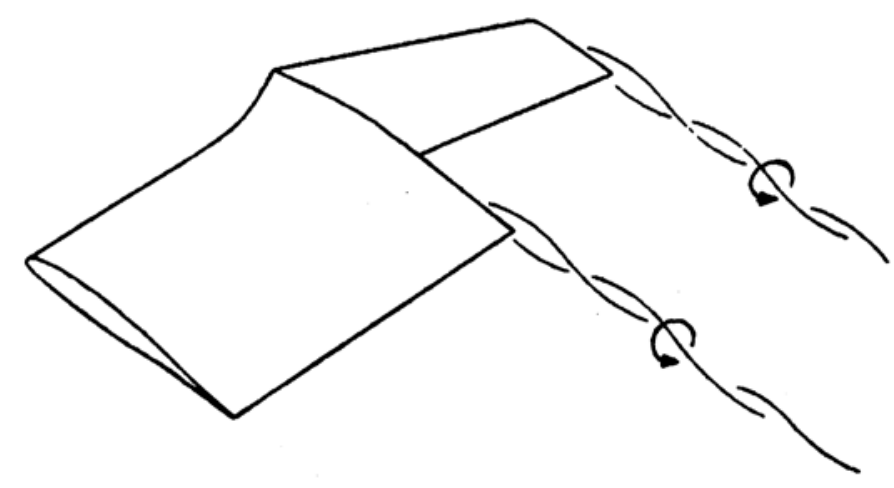

Fig. 1. Vane tip configuration with trailed twin vortices (taken from Ref. 22) 


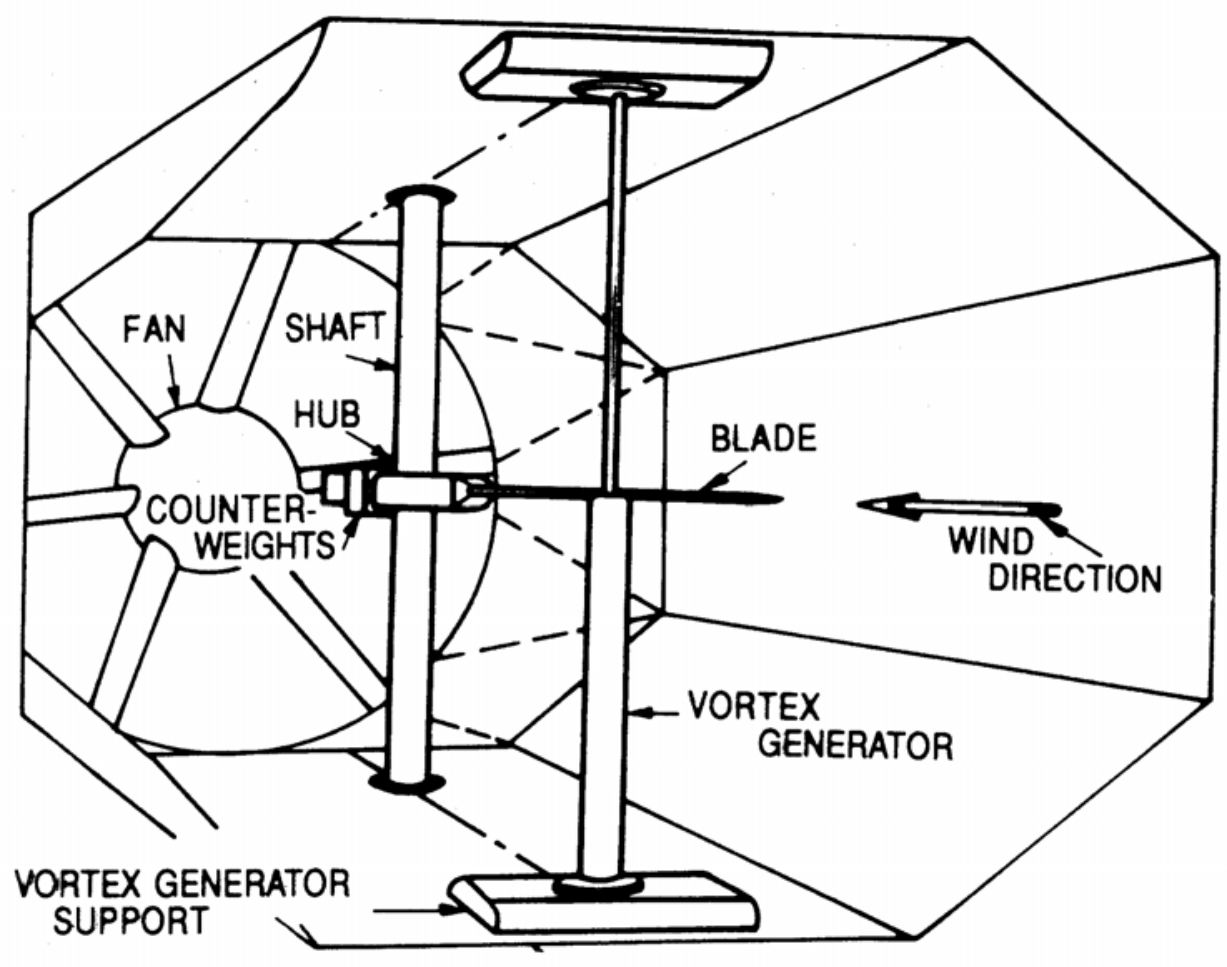

Fig. 2. Illustration of the Glasgow University blade-vortex interaction facility 

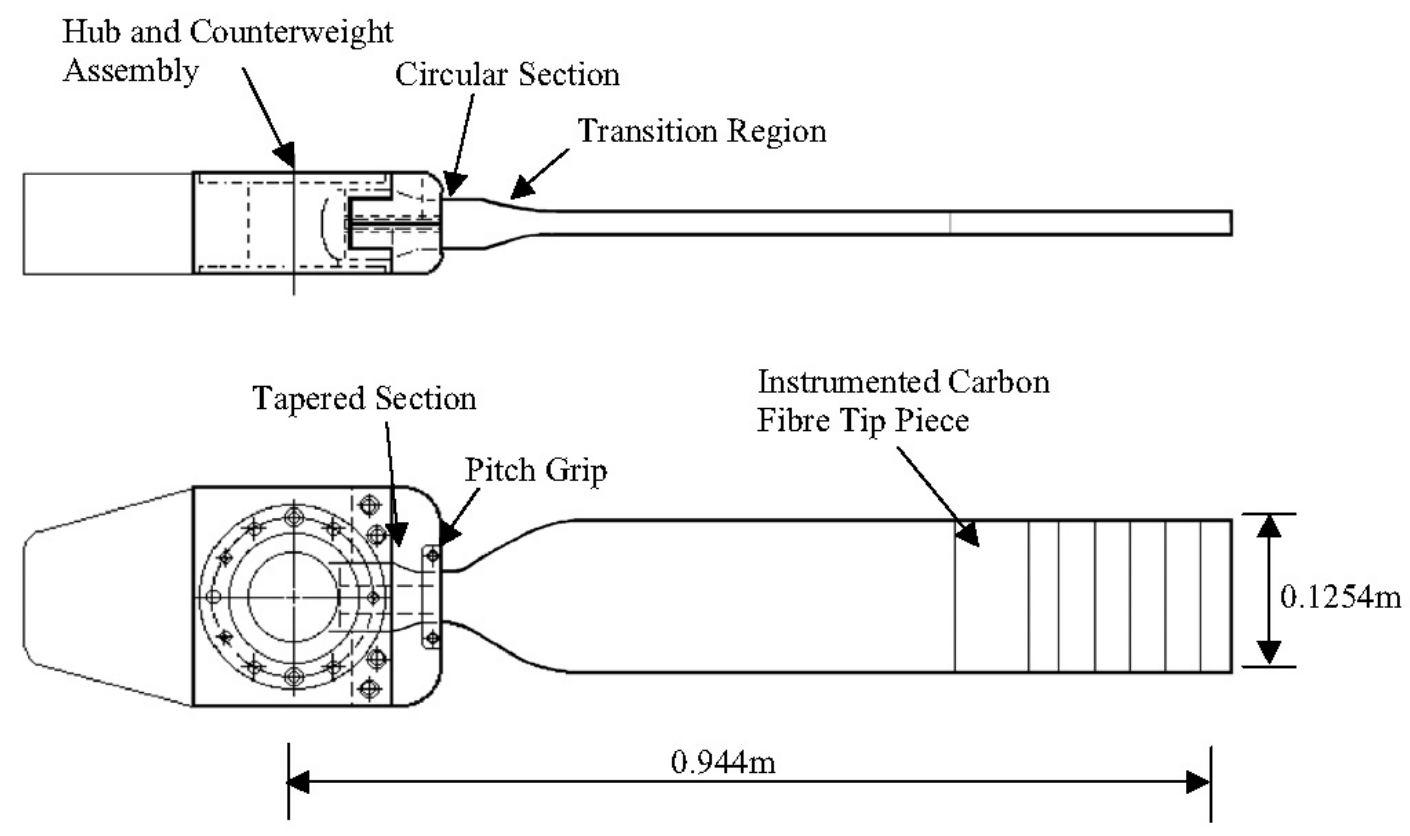

Fig. 3. Construction of rotor blade 


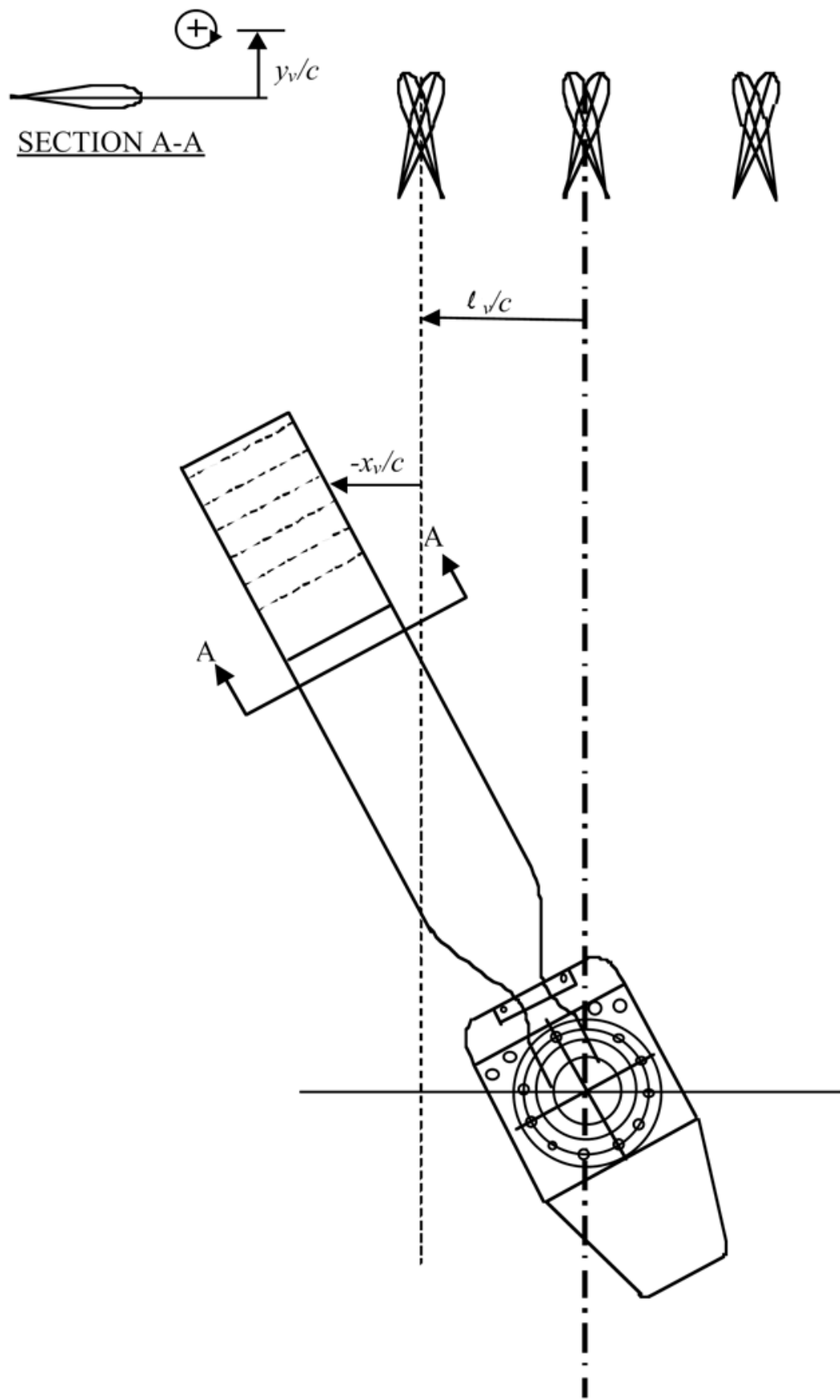

Fig. 4. Geometric parameters defining BVI settings 


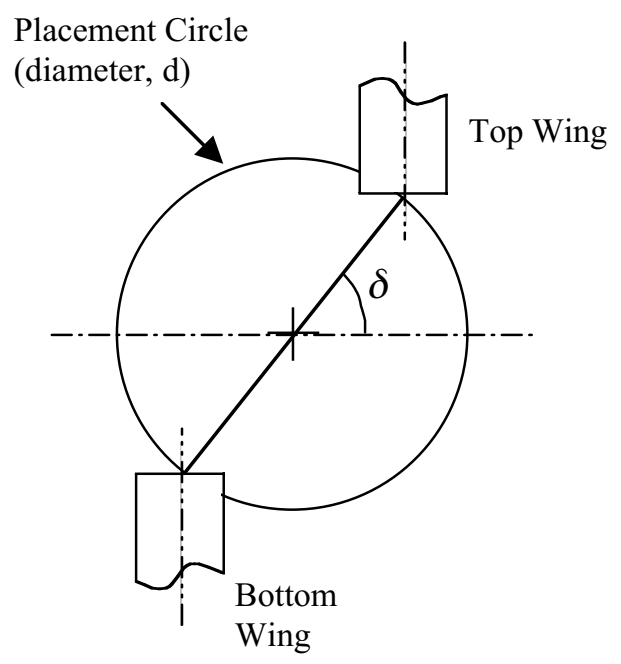

Fig. 5. Twin vortex generator geometry 


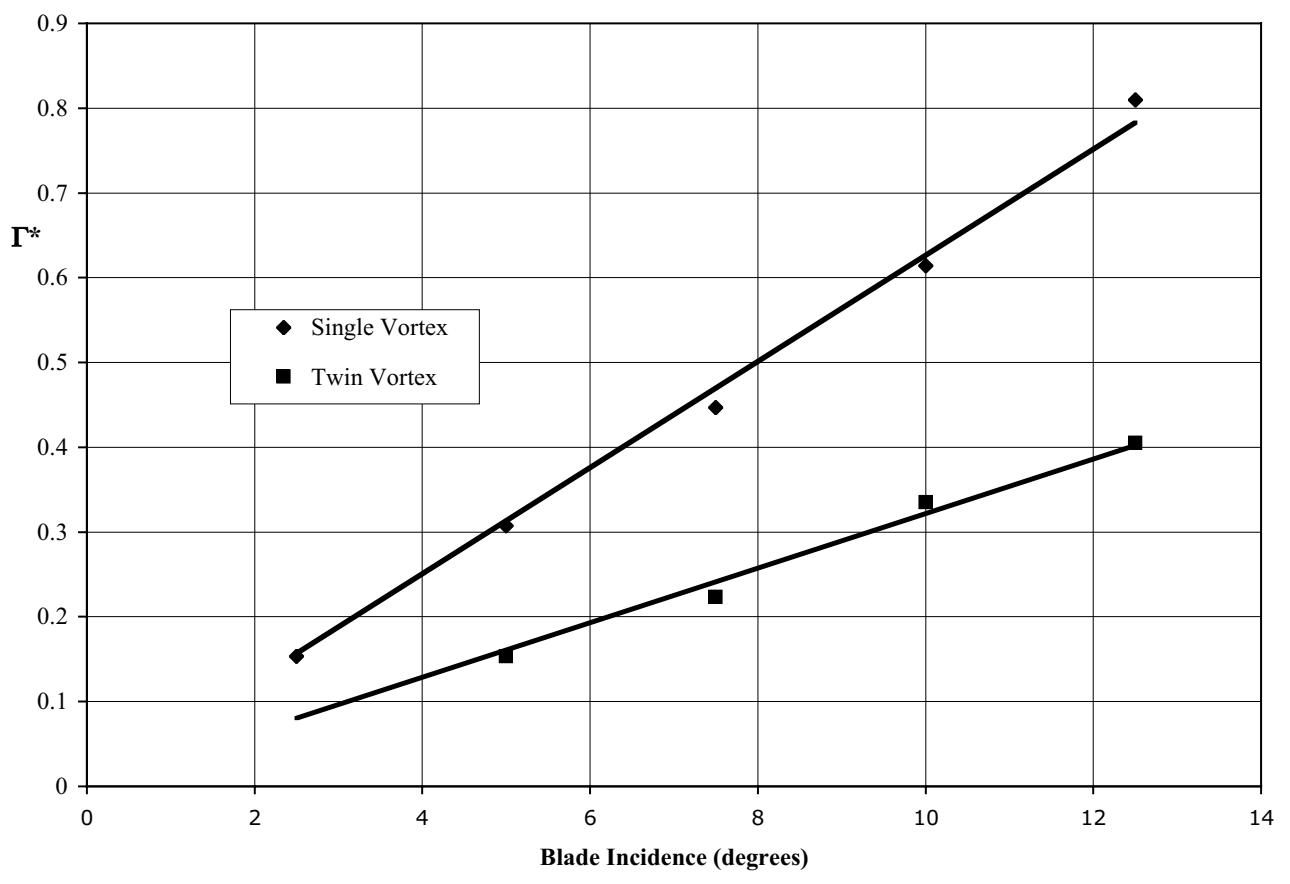

Fig. 6. Variation of single and twin vortex strength with generator blade incidence. Twin vortex $d / c=0.5$. 


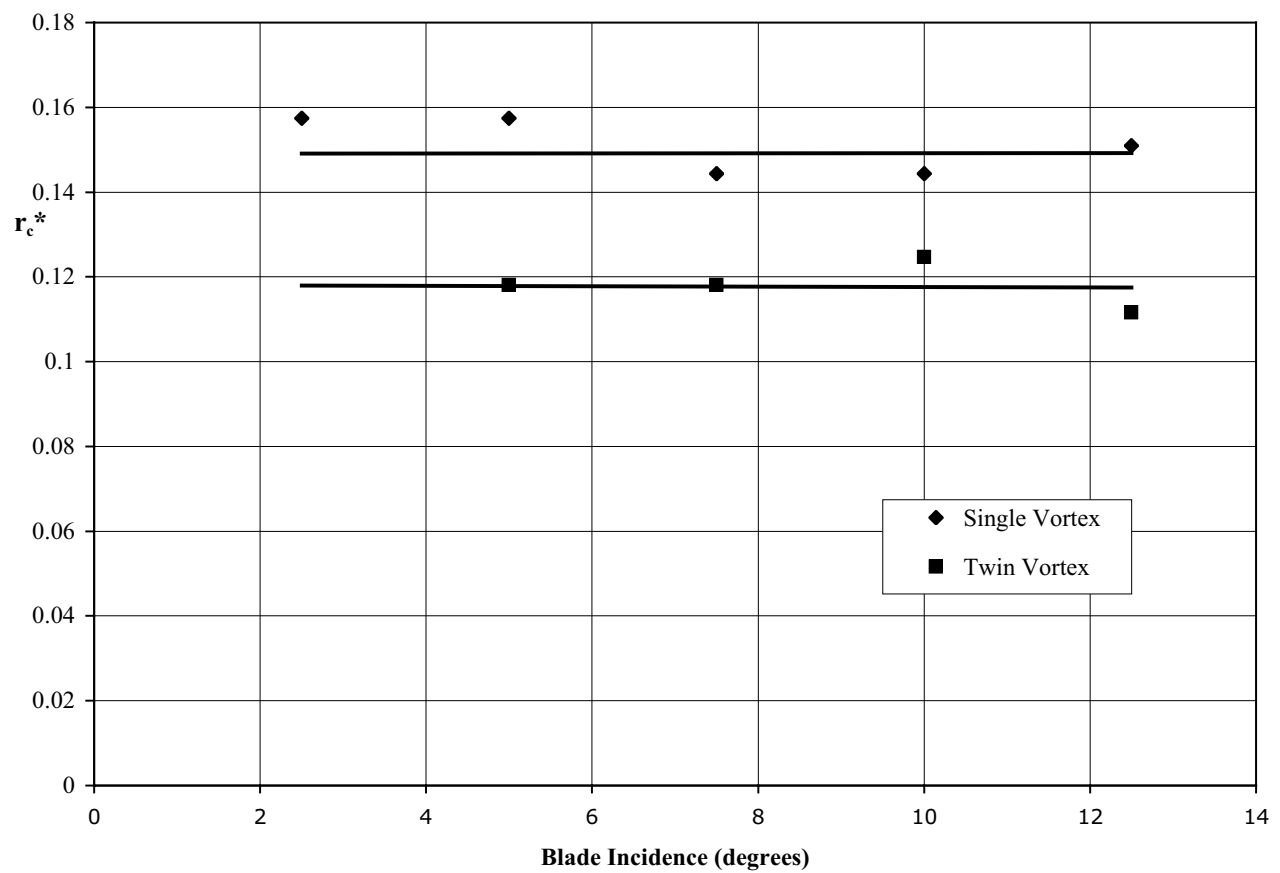

Fig. 7. Variation of twin and single vortex core size with generator blade incidence.

Twin vortex $d / c=0.5$. 


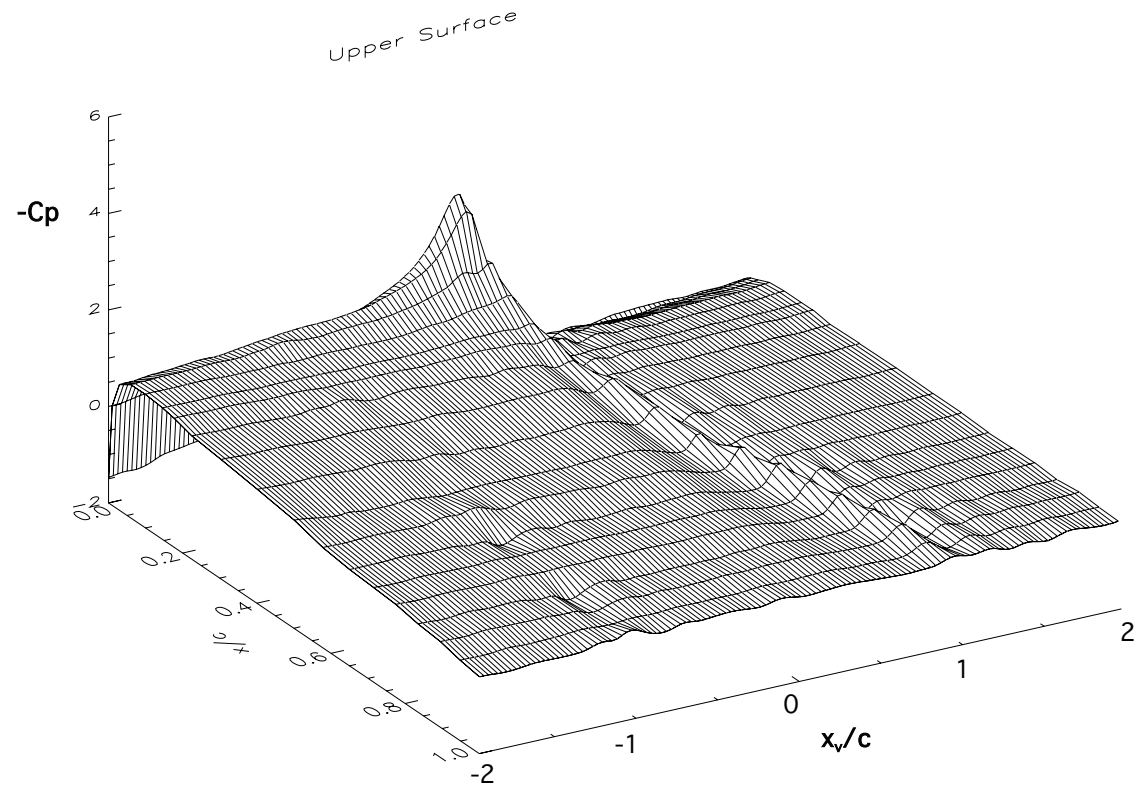

Fig. 8. Upper surface pressure variation for a parallel interaction with a single vortex

$$
\left(\Gamma^{*}=0.81, \Gamma_{I}=0.641, \ell_{v} / c=0, y_{v} / c=0, r / R=0.785 \theta=0^{\circ}\right)
$$




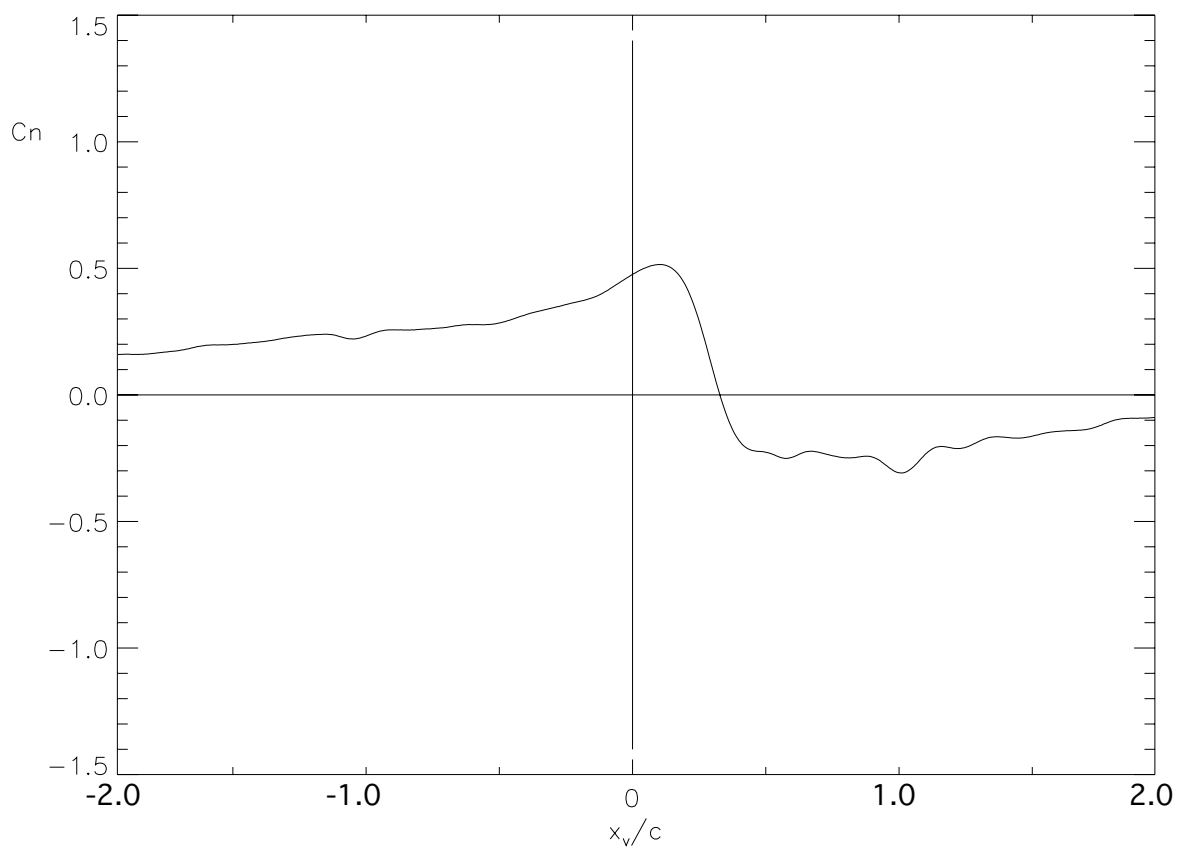

Fig. 9. Normal force coefficient variation during a parallel interaction with a single $\operatorname{vortex}\left(\Gamma^{*}=0.81, \Gamma_{I}=0.641, \ell_{v} / c=0, y_{v} / c=0, r / R=0.785 \theta=0^{\circ}\right)$ 


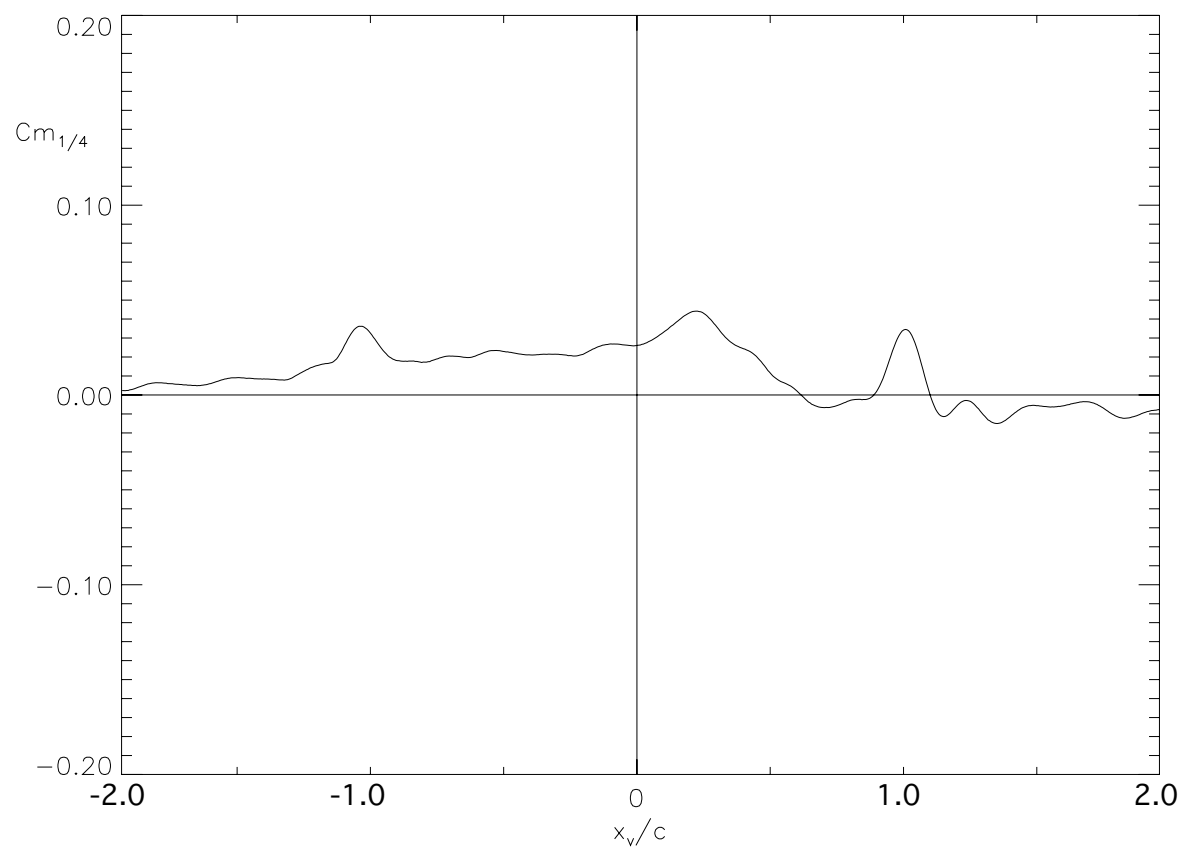

Fig. 10. Pitching moment coefficient variation during a parallel interaction with a single $\operatorname{vortex}\left(\Gamma^{*}=0.81, \Gamma_{I}=0.641, \ell_{v} / c=0, y_{v} / c=0, r / R=0.785 \theta=0^{\circ}\right)$ 


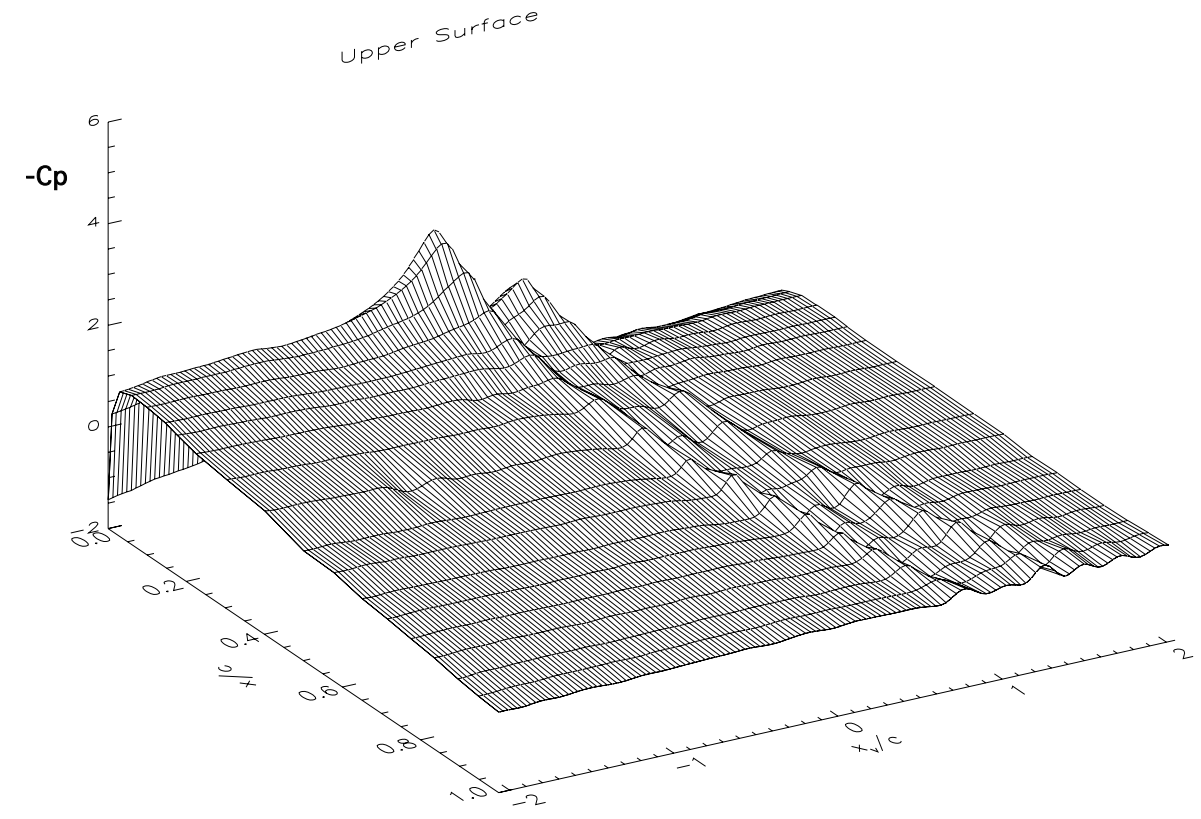

Fig. 11a. Upper surface pressure variation for a parallel interaction with a twin vortex system of combined strength $\Gamma^{*}=0.81, \Gamma_{I}=0.641$. Cycle 1 of $16\left(\mathrm{~d} / \mathrm{c}=0.47, \delta=0^{\circ}\right.$, $\left.\ell_{v} / c=0, y_{v} / c=0, r / R=0.785 \theta=0^{\circ}\right)$ 


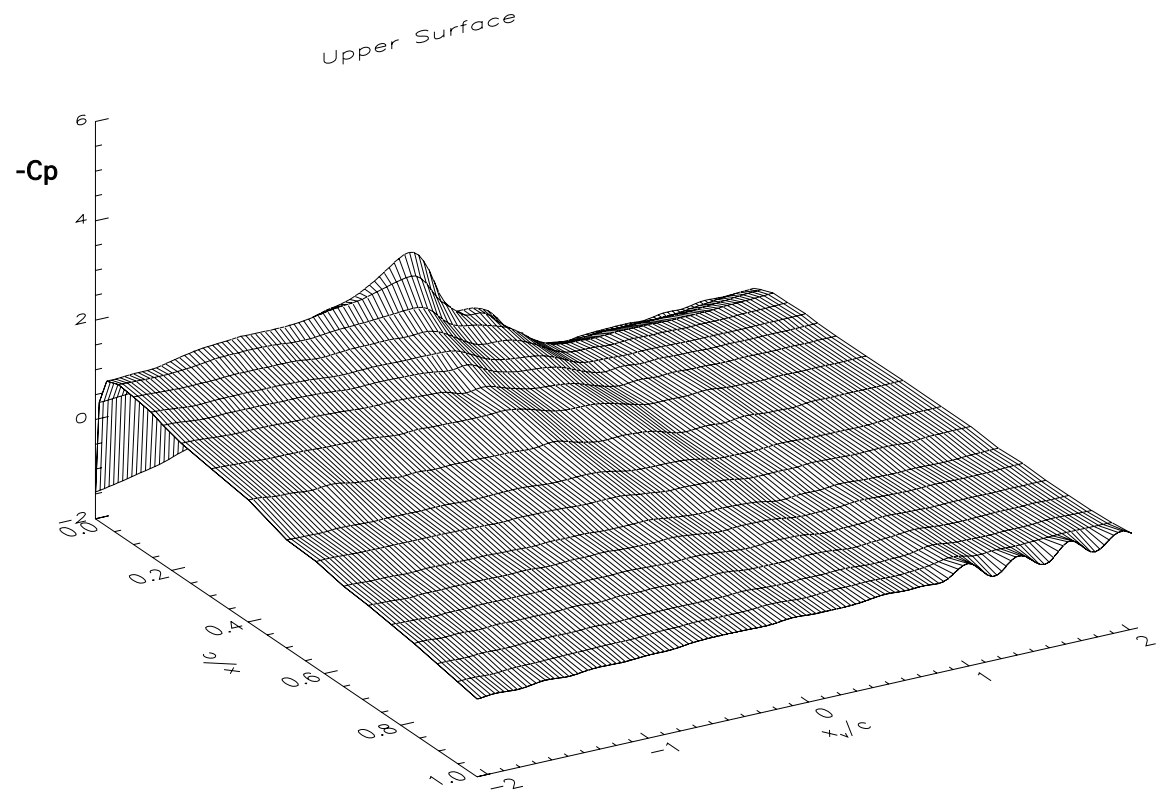

Fig. 11b. Upper surface pressure variation for a parallel interaction with a twin vortex system of combined strength $\Gamma^{*}=0.81, \Gamma_{I}=0.641$. Cycle 10 of $16\left(\mathrm{~d} / \mathrm{c}=0.47, \delta=0^{\circ}\right.$,

$$
\left.\ell_{v} / c=0, y_{v} / c=0, r / R=0.785 \theta=0^{\circ}\right)
$$




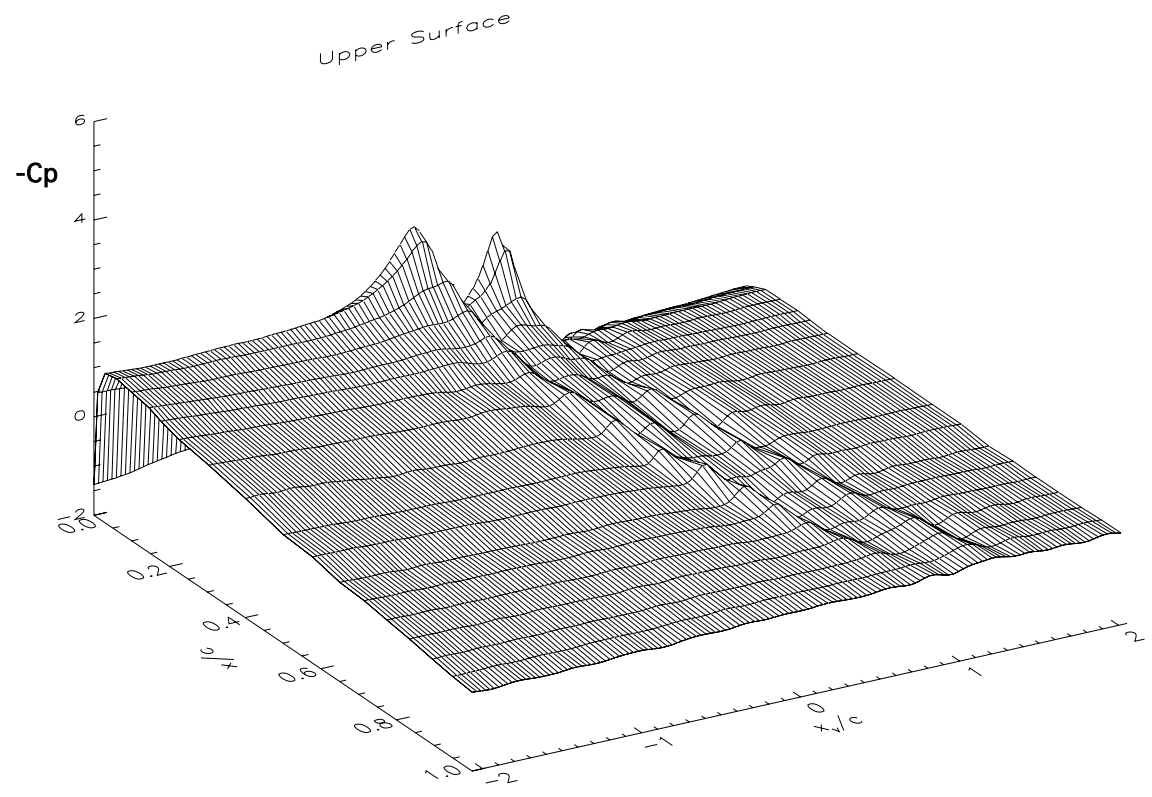

Fig. 11c. Upper surface pressure variation for a parallel interaction with a twin vortex system of combined strength $\Gamma^{*}=0.81, \Gamma_{I}=0.641$. Cycle 13 of $16\left(\mathrm{~d} / \mathrm{c}=0.47, \delta=0^{\circ}\right.$,

$$
\left.\ell_{v} / c=0, y_{v} / c=0, r / R=0.785 \theta=0^{\circ}\right)
$$




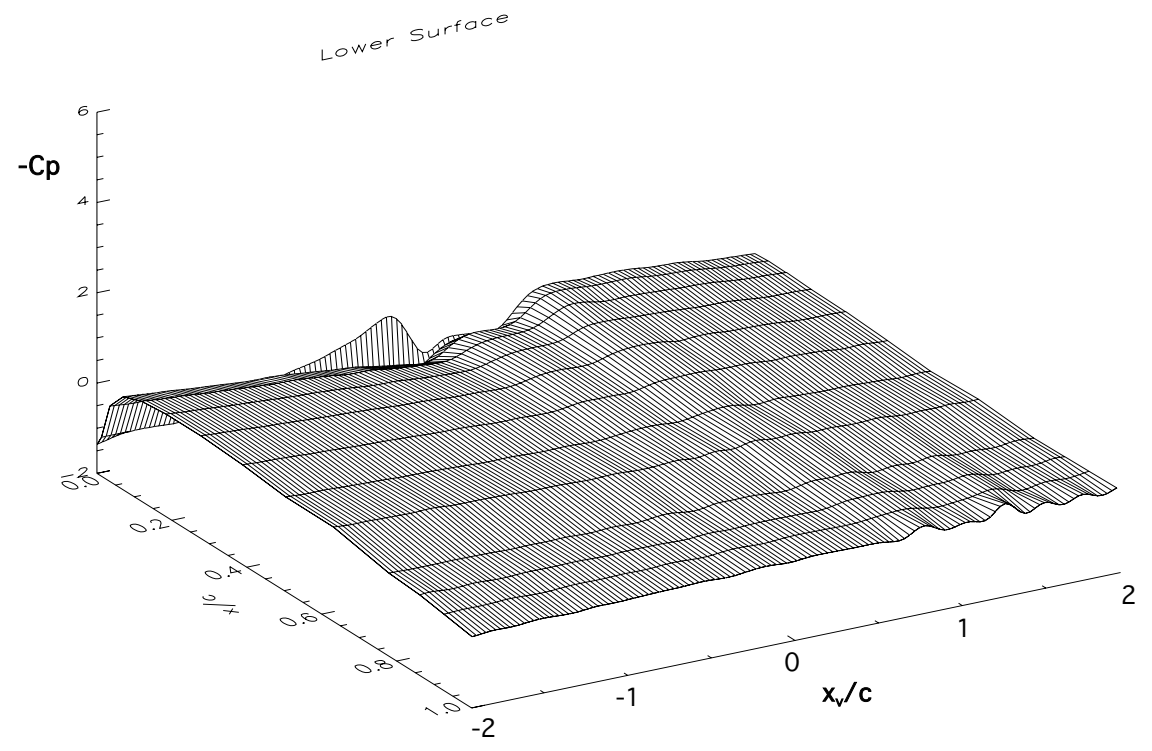

Fig. 12a. Lower surface pressure variation for a parallel interaction with a twin vortex system of combined strength $\Gamma^{*}=0.81, \Gamma_{I}=0.641$. Cycle 1 of $16\left(\mathrm{~d} / \mathrm{c}=0.47, \delta=0^{\circ}\right.$, $\left.\ell_{v} / c=0, y_{v} / c=0, r / R=0.785 \theta=0^{\circ}\right)$ 


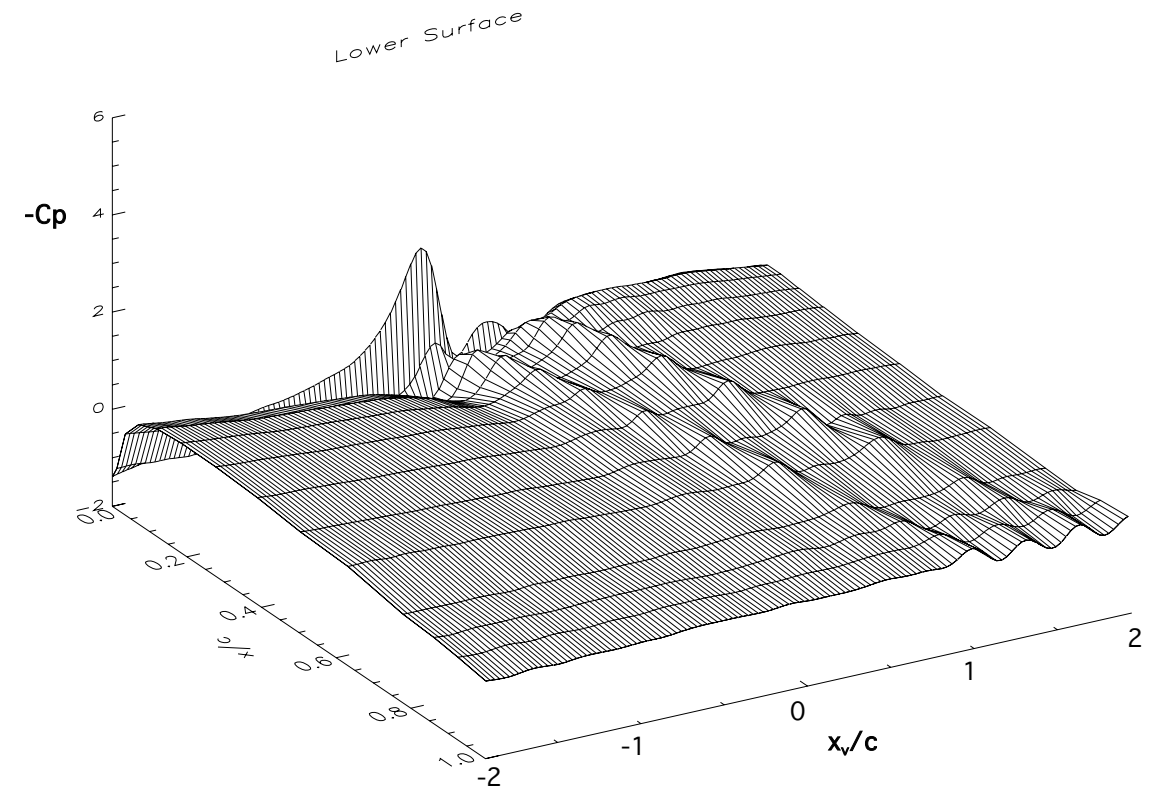

Fig. 12b. Lower surface pressure variation for a parallel interaction with a twin vortex system of combined strength $\Gamma^{*}=0.81, \Gamma_{I}=0.641$. Cycle 10 of $16\left(\mathrm{~d} / \mathrm{c}=0.47, \delta=0^{\circ}\right.$,

$$
\left.\ell_{v} / c=0, y_{v} / c=0, r / R=0.785 \theta=0^{\circ}\right)
$$




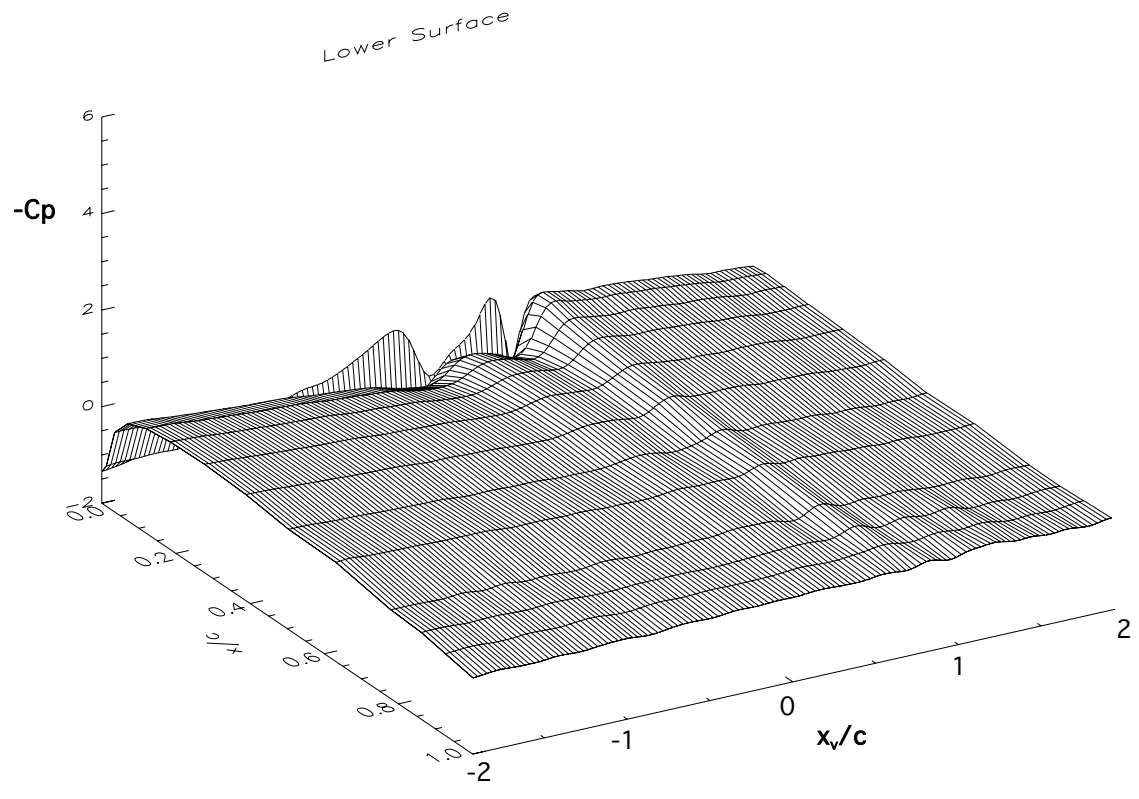

Fig. 12c. Lower surface pressure variation for a parallel interaction with a twin vortex system of combined strength $\Gamma^{*}=0.81, \Gamma_{I}=0.641$. Cycle 13 of 16( $\left.\mathrm{d} / \mathrm{c}=0.47, \delta=0^{\circ}, \ell_{v} / c=0, y_{v} / c=0, r / R=0.785 \theta=0^{\circ}\right)$ 


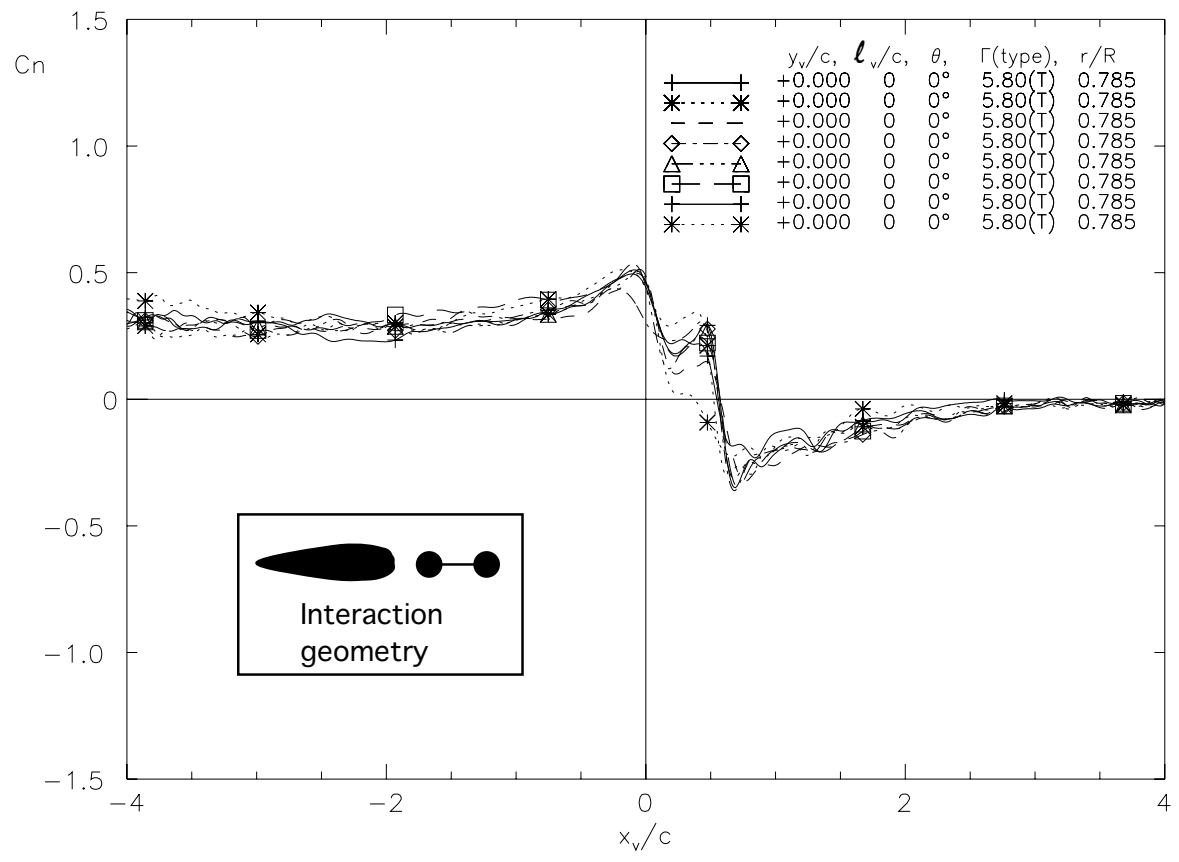

Fig. 13a Normal force coefficient variation during a parallel interaction with a twin vortex system of combined strength $\Gamma^{*}=0.81, \Gamma_{I}=0.641 .\left(\mathrm{d} / \mathrm{c}=0.47, \delta=0^{\circ}, \ell_{v} / c=0\right.$,

$$
\left.y_{v} / c=0, r / R=0.785 \theta=0^{\circ}\right)
$$




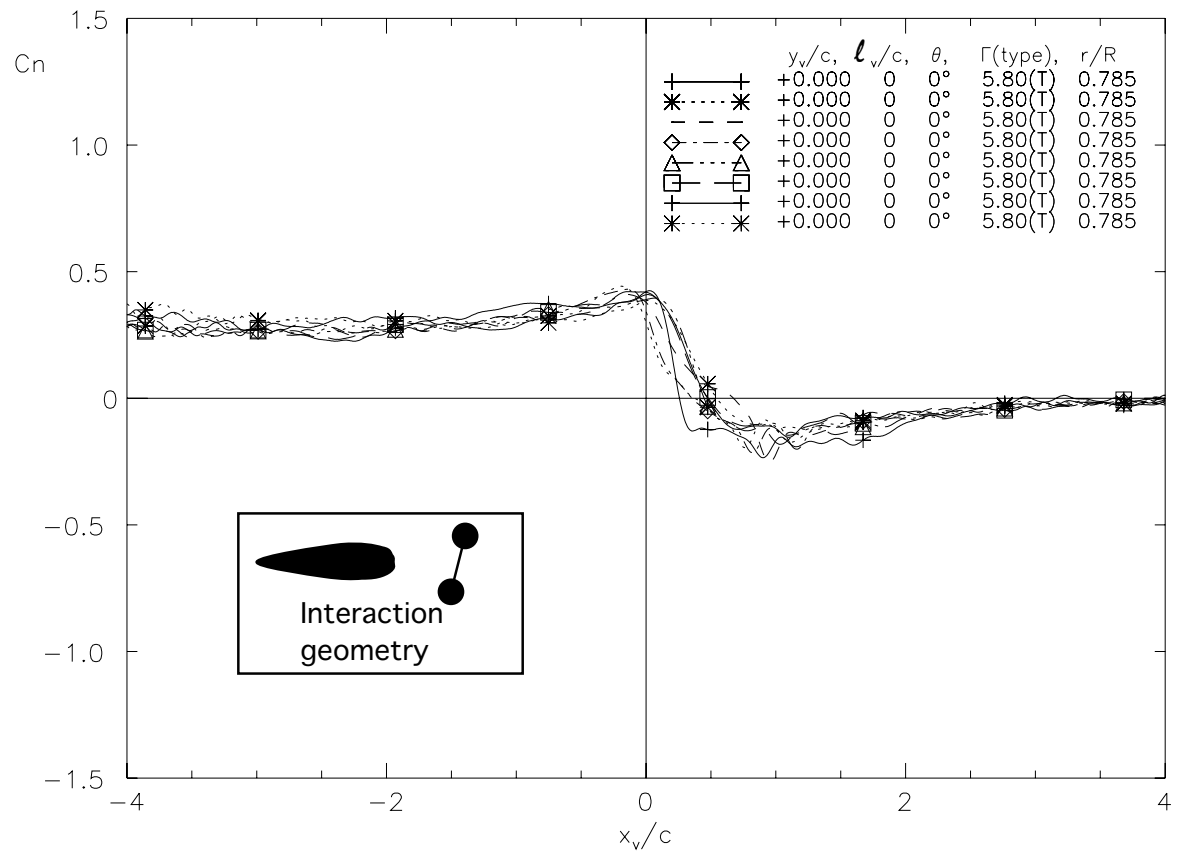

Fig. 13b Normal force coefficient variation during a parallel interaction with a twin vortex system of combined strength $\Gamma^{*}=0.81, \Gamma_{I}=0.641 .\left(\mathrm{d} / \mathrm{c}=0.49, \delta=75.41^{\circ}, \mathrm{lv}_{v} / \mathrm{c}\right.$

$$
=0, y_{v} / c=0, r / R=0.785 \theta=0^{\circ} \text { ) }
$$




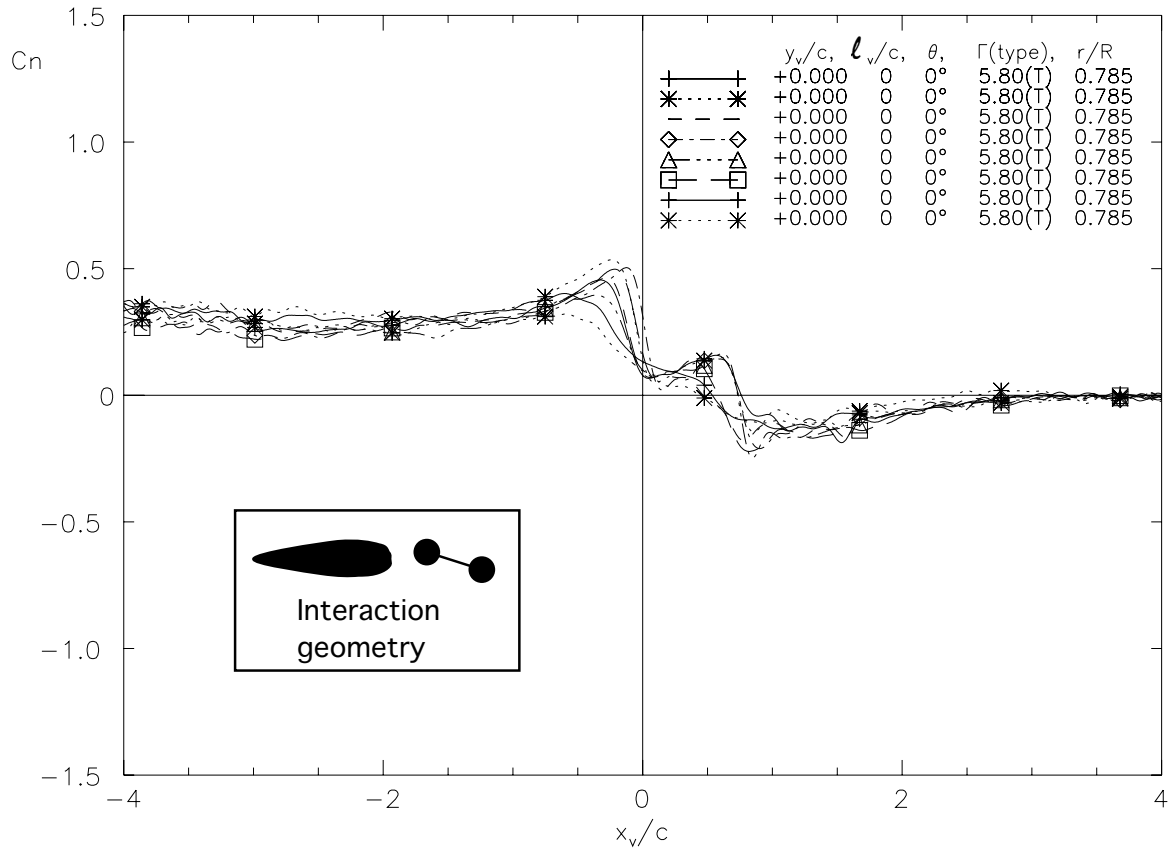

Fig. 13c Normal force coefficient variation during a parallel interaction with a twin vortex system of combined strength $\Gamma^{*}=0.81, \Gamma_{I}=0.641 .\left(\mathrm{d} / \mathrm{c}=0.79, \delta=161.71^{\circ}\right.$,

$$
\left.\ell_{v} / c=0, y_{v} / c=0, r / R=0.785 \theta=0^{\circ}\right)
$$




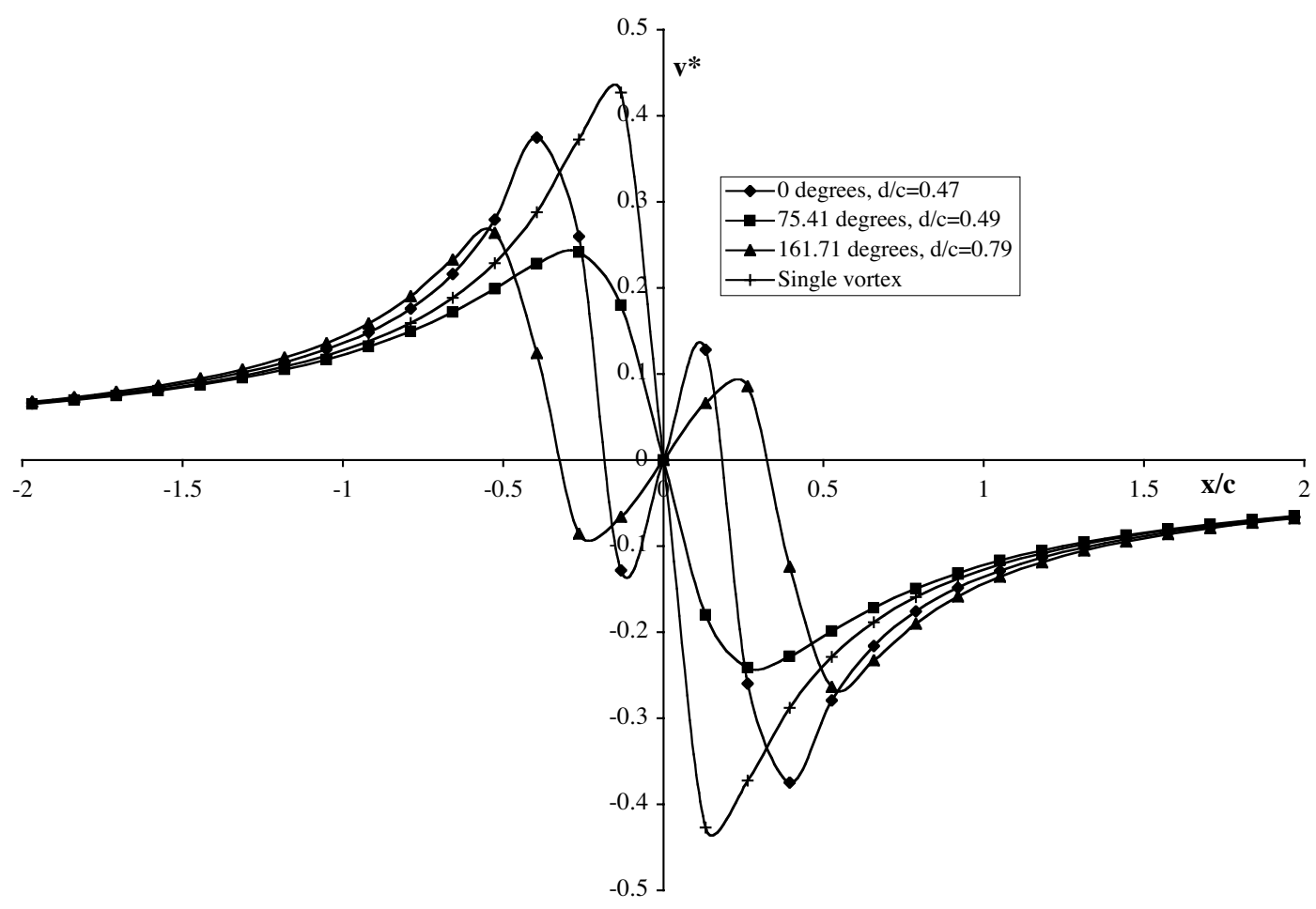

Fig. 14. Calculated vertical velocity distribution for single vortex and three twin vortex cases of combined strength $\Gamma^{*}=0.81$. 


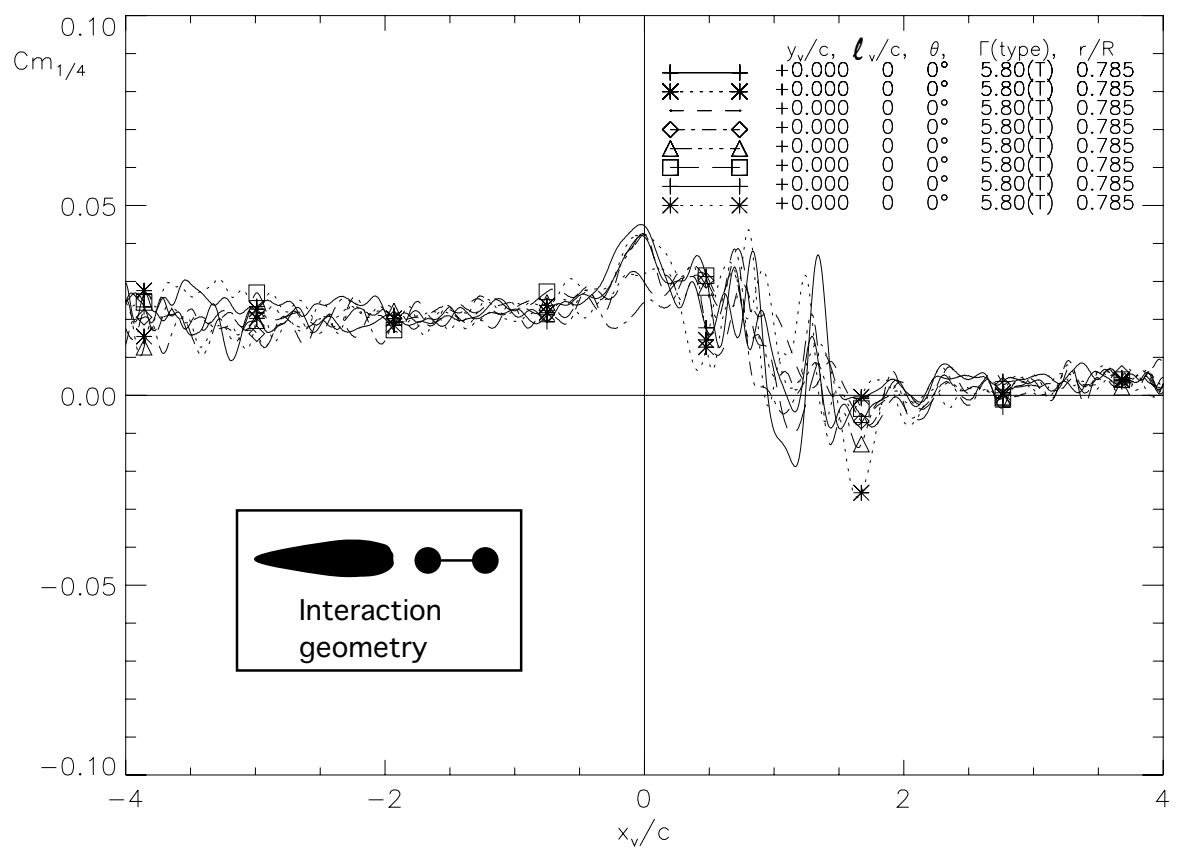

Fig. 15 Pitching moment coefficient variation during a parallel interaction with a twin vortex system of combined strength $\Gamma^{*}=0.81, \Gamma_{I}=0.641 .\left(\mathrm{d} / \mathrm{c}=0.47, \delta=0^{\circ}, \ell_{\mathrm{l}} / \mathrm{c}=0\right.$,

$$
\left.y_{v} / c=0, r / R=0.785 \theta=0^{\circ}\right)
$$

